\title{
A review of ideas and strategies to improve scientific research and its dissemination in life and social sciences
}

Hannes Almgren ${ }^{1, *}$, Nicholas Parsons ${ }^{2}$, Sofie Van Den Bossche ${ }^{1}$, Daniele Marinazzo ${ }^{1}$

1 Department of Data Analysis, Faculty of Psychology and Educational Sciences, Ghent University, Belgium

${ }^{2}$ Cognitive Neuroscience Unit, School of Psychology, Deakin University, Melbourne Burwood Campus, VIC, Australia

*Corresponding author: Hannes.Almgren@ugent.be 


\section{Table of contents}

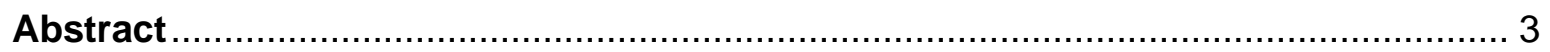

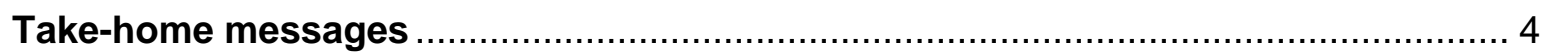

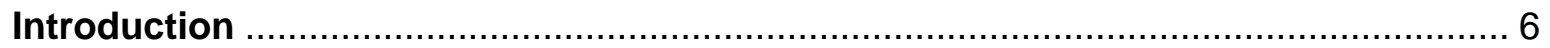

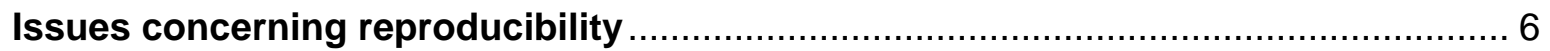

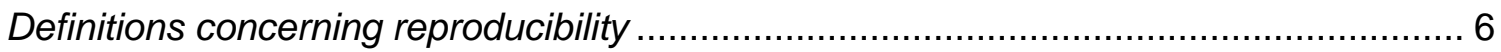

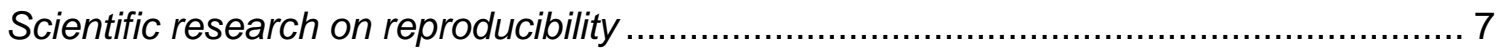

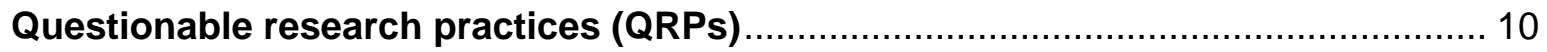

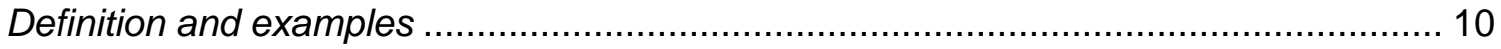

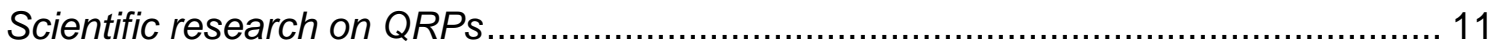

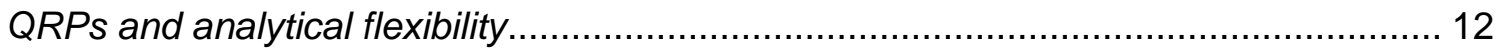

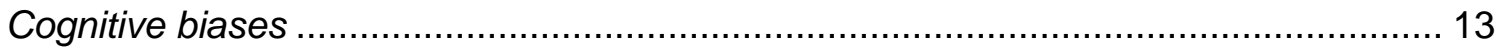

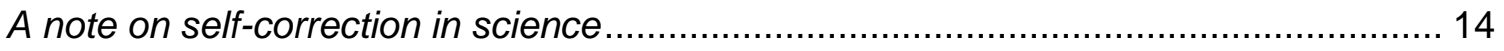

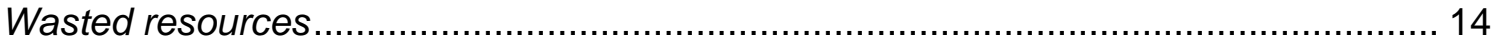

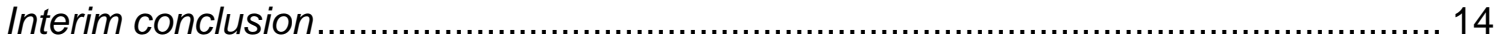

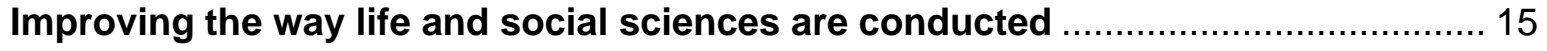

Improvement 1: Focus on aggregate effects across replication attempts .................... 16

Improvement 2: Improved preparation and achieving more solid empirical claims

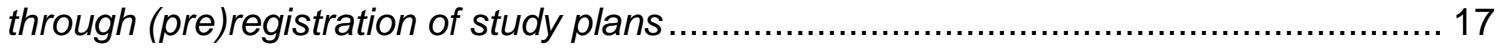

Improvement 3: Expanding our statistical toolbox and justifying analytical choices a

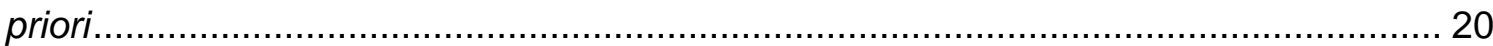

Improvement 4: Transparency, complete reporting, and openness (if warranted) of data, code, all performed analyses, results, and (changes in) research plans ....................... 24

Improvement 5: Improving the way we decide what is considered scientific ................. 28 Improvement 6: Changing incentives in science from a focus on quantity to quality and

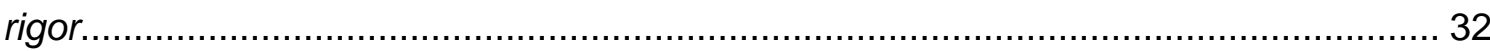

In perspective: Science as a social enterprise.................................................... 36

Crowdsourcing and horizontal models of science .................................................. 36

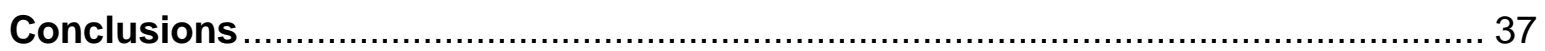

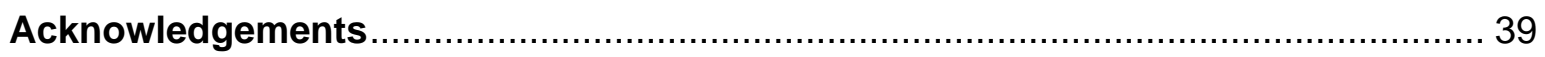

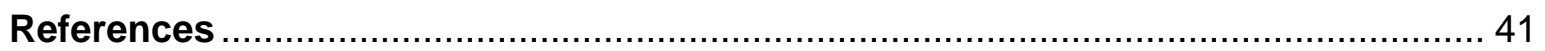




\section{Abstract}

In recent years, issues concerning reproducibility, questionable research practices (QRPs), and analytical flexibility have been contentious topics in the fields of life and social sciences. As such, research has increasingly focused on studying the way we conduct science (which is a field of study in meta-science), and new ideas have been introduced on how we can improve the way we conduct science. In this literature review, we aim to summarize research and ideas that have been introduced in the past decade. We give an overview of studies concerning reproducibility in contemporary life and social sciences, summarize reasons for failed and successful reproductions, and outline QRPs which are often used when results do not support the researcher's hypothesis and/or when results are not statistically significant. We also discuss cognitive biases in research and the topic of analytical flexibility, which warrants straightforward interpretation and generalization of results. We summarize the literature on improving the scientific process and system of life and social sciences in six broad categories, including (1) Focus on aggregate effects across replication attempts to achieve scientific knowledge, (2) Improved preparation and achieving more solid empirical claims through (pre)registered reports when performing hypothesis-driven research, (3) Expanding our statistical toolbox by going beyond null-hypothesis significance testing, and justifying analytical choices a priori, (4) Transparency, complete reporting, and openness (if warranted) of data, code, all performed analyses, results, and (changes in) research plans, (5) Improving the way we decide what is considered scientific by allowing for continuous, graded, open, and (optionally anonymous) peer review, and by giving reviewers checklists, and (6) Changing incentives in science from a focus on quantity to quality and rigor by focusing on research metrics that more closely reflect scientific quality (e.g., by using graded peer evaluations). 


\section{Take-home messages}

\section{Recent research has shown that:}

- An important factor in reproducibility is the prior odds of the hypothesis in question being true, which is field-dependent (Wilson \& Wixted, 2018):

$\circ$ Deducing hypotheses from theory might increase their prior odds (Fiedler, 2017).

- Effect sizes are often over-estimated in articles that are not (pre)registered, which also causes over-estimation of meta-analytical effect sizes (Kvarven et al., 2020).

- Scientific self-correction is currently suboptimal.

- Researchers should make sure they do not overemphasize positive results in the literature if there is no methodological or statistical reason to do so.

- Researchers should be critical about the studies they cite (Bordignon, 2020).

- Questionable research practices are often applied, especially when the tested hypothesis is not supported by the data (see, e.g. Artino et al., 2019).

- A downside of preprints is the potential dissemination of low-quality research (e.g., when conclusions that are not supported by the data; Glasziou et al., 2020; Besançon et al., 2020), as well as research with financial and/or political motives. Researchers should be vigilant and report such cases.

- P-values are difficult to interpret in exploratory research (Nosek \& Lakens, 2014).

- Most, if not all, researchers have conflicts of interests, which include maintaining or building a career (Munafò et al., 2017). These implicit COls might have a substantial impact on research, though this has not yet been investigated often. 


\section{Recent ideas to improve social and life sciences include:}

- Continuous, graded and open rating of scientific articles' qualities by peers.

- E.g., using social web interfaces (Yarkoni, 2012)

- Incentivization of (open) high quality peer reviews by making them open, citable, and/or paying reviewers, with optional anonymity (see e.g., Mambrini et al., 2020; Tracz \& Lawrence, 2016).

- Journals, articles, and other research material could be evaluated using new and more refined standards, for example based on methodological rigor, transparency/openness, and clarity, amongst many others (see e.g., Woolston, 2020; Yarkoni, 2012).

- Peer reviews could become (partly) more objective by giving peer reviewers checklists (see e.g., Nosek et al., 2012).

- Researchers should focus more on effect sizes (with corresponding confidence intervals), as well as use alternatives to null-hypothesis testing when more applicable (see e.g., Cumming, 2014, McShane et al., 2019).

- Sometimes complete openness may be unwarranted, especially when dealing with highly sensitive data and/or results. In such case, transparency regarding how to access the research material (including specific instructions how to access) is important.

- New generations of scientists should be trained extensively in acceptable research conduct, statistics, methodology, and theory development (amongst others) without too much pressure to publish. 


\section{Introduction}

Issues concerning reproducibility, questionable research practices, as well as openness (and transparency) in science, have been heavily discussed over the past decade. In some scientific fields, such as tumor biology and psychology, there is increasing focus on estimating reproducibility. These estimates range from low (see, e.g., Prinz et al., 2011), to moderate or high (see, e.g., Klein et al., 2014). Other research focuses on questionable research practices, which are harmful but ubiquitous scientific practices; likely effecting the validity of scientific evidence (Artino et al., 2019; Banks et al., 2016).

In the past decade, new ideas have been introduced to improve the way we conduct research in life and social sciences. In their series called 'scientific utopia', Brian Nosek and colleagues introduced new ways of scientific communication (Nosek \& Bar-Anan, 2012), ideas concerning restructuring incentives in science (Nosek et al., 2012), and the idea of crowdsourcing and large-scale collaborations in science (Uhlmann et al., 2019). In a series on 'reducing waste and increasing value' in biomedical science, researchers outlined the issues of (amongst others) wasted scientific resources (e.g., money) and inaccessible but essential study-related information in biomedical research (see, e.g., Chan et al., 2014; Macleod et al., 2014). Recent papers in neuroscience (e.g., Gorgolewski et al., 2016; Gorgolewski \& Poldrack, 2016; Nichols et al., 2017) disseminated modern platforms, open software, and ideas to improve the way computational and cognitive neuroscience is conducted. In 2017, Munafò and colleagues wrote a manifesto for reproducible science, which outlined measures that could improve transparency, reproducibility, and efficiency in research. In 'Psychology's Renaissance', Nelson at al. (2018) reviewed the issues and changes in psychological science in the past decade. More recently, Besançon et al. (2020) discussed the (mis)use of open scientific principles in COVID-19 research, which has been signed by 371 people (mainly researchers and medical doctors).

The goal of this (meta-)review is to provide a balanced and broad up-to-date literature review of past and recent meta-research, previous reviews, and new ideas concerning how we can improve the way science is conducted across life and social sciences. The content of this paper is divided as follows: First we summarize the issues concerning reproducibility across disciplines, next we focus on practices that might have caused issues concerning reproducibility (namely questionable research practices), we proceed by summarizing the literature in six broad categories of (potential) solutions to improve the way science is done across multiple disciplines in life and social sciences, and we take a perspective on science as a social enterprise.

\section{Issues concerning reproducibility}

\section{Definitions concerning reproducibility}

It is important to clarify how different concepts related to reproducibility are defined; unfortunately, there is little consensus on the terminology used (Plesser, 2017). Goodman et al. (2016) divided reproducibility in three categories: methods, results, and inferential reproducibility, yet Nichols et al. (2017) suggested a more fine-grained categorization tailored to a specific field (here: functional MRI neuroimaging), while others use alternate definitions 
(see, e.g., Whitacker, 2008). Moreover, the same terms have been used differently by different researchers (Plesser, 2017). In the present article, we will use the three general categories outlined in Goodman et al. (2016): methods reproducibility, results reproducibility, and inferential reproducibility. Methods reproducibility refers to the possibility to reproduce the methods outlined in a scientific article, for example by having enough detail about the procedures and analyses. This might include, amongst others, dissemination of code for the analyses, and dissemination of data. Results reproducibility refers to the reproduction of the results by a different study that closely matches the original study. Methods reproducibility does not imply results reproducibility even when the same data is used, since sometimes even different computer operating systems can contribute to variation in results (see, e.g., Glatard et al., 2015). Inferential reproducibility refers to the reproduction of the same conclusions by a different study (e.g., by using different data and/or different methods), which is often difficult to quantify. In this work we use the words replicability and reproducibility synonymously.

In general, it may be good practice to be specific about which variables are kept constant between the original and replication studies (i.e., what aspects of the data, analyses, and procedures), as well as to be specific about what exactly was reproduced, for example the specific part of the methods, results, and/or conclusion (for an example in neuroimaging, see Nichols et al., 2017).

\section{Scientific research on reproducibility}

\section{Cancer biology}

Though a successful reproduction is difficult to define (Nosek \& Errington, 2017), estimates of reproducibility across scientific disciplines are often low. Studies on reproducibility of clinical and preclinical cancer studies showed that only between $11 \%$ and $25 \%$ of studies could be reproduced (Begley \& Ellis, 2012; Prinz et al., 2011), though it is ambiguous which studies failed to reproduce results compared to methods. Note that these studies did not report details about the performed studies which makes it difficult to evaluate them critically. In a more recent reproducibility project of studies on cancer biology, results of five cancer biology studies were attempted to be reproduced. Results of one study could not be reproduced, while two reproductions were uninterpretable (e.g., because of problems with tests to measure tumor growth; Baker \& Dolgin, 2017, which can be categorized as failed methods reproducibility; see Goodman et al., 2016), and two studies were substantially reproduced. The same article argued that the clearest finding was that many papers do not provide sufficient detail about their methods. Note that the same results have been interpreted more negatively by Wen et al. (2018), who classified four out of the five studies as not reproduced, and one as uninterpretable. Eisner (2018) gave some suggestions on the reasons why some biomedical research is difficult to be reproduced, including the pressure to publish in high impact journals but also technical factors such as incorrectly labelled cell lines and low power.

It has been estimated that the annual costs of preclinical research that is not reproducible is around 28 billion US dollars in the United States of America (Freedman et al., 2015). Moreover, 107 cancer biology studies were retracted in 2017 because of fake peer reviews (Stigbrand, 2017), which may reflect deeper issues surrounding scientific conduct. Issues with reproducibility have also been identified in medicine, though often it is not referred to as reproducibility (see, e.g., Prasad et al., 2013). 


\section{Psychology and social sciences}

If issues concerning reproducibility exist in vital scientific areas like cancer biology, how reproducible are studies in other (potentially less vital) scientific areas? Research on reproducibility in psychology showed that only one-third to one-half of results could be reproduced (Open Science Collaboration, 2015). Results of cognitive psychology studies were more replicable compared to results of social psychology studies, which has been attributed to differences in power as well as prior odds of the effect being true (Wilson \& Wixted, 2018; Open Science Collaboration, 2015). The same study (i.e., Open Science Collaboration, 2015) also reported that the reproduced effect sizes were half of the magnitude of the original effect sizes. Though, a response to this paper included criticism for not adhering to the original populations, and for only re-conducting each experiment once (Gilbert et al., 2016). In another study, Klein et al. (2014) assessed the reproducibility of thirteen psychological effects published in scientific articles. Thirty-six research teams acquired data using exactly the same experiment. They found that the results of the majority of studies $(77 \%)$ were reproduced. A more recent study (Camerer et al., 2018) showed that results of about $57 \%$ to $67 \%$ (depending on the replication indicator) of 21 experimental studies in social sciences could be reproduced. These reproduced effect sizes were on average $46 \%$ of the magnitude of effect sizes reported by the original studies.

\section{Cognitive neuroscience}

In cognitive neuroscience, a recent article invoked 70 independent teams of scientists to test the same nine hypotheses using an openly available dataset (Botvinik-Nezer et al., 2020). It is important to stress that the same dataset was analyzed by all researchers. Little consistency between the research teams was found for five hypotheses, highly consistent statistically significant findings were found for only one hypothesis, while three hypotheses were found to be statistically insignificant by most teams (note that this is a reproduction of statistically insignificant results). Their dataset contained 108 subjects (54 subjects in each group), which is considered high compared to sample sizes in most functional MRI studies (Poldrack et al., 2017; Szucs \& loannidis, 2017a). This study showed variability in about half of the results using the same data; most likely attributable to differences in neuroimaging processing steps and software packages. The authors suggested the use of meta-analysis across processing pipelines as a potential solution for discrepancies between research teams, which is discussed in our paragraph on aggregate effects across replication attempts. This study also found that neuroscientists overestimate the probability of significant effects, which was shown using prediction markets in which researchers bet on whether results would be replicated (Dreber et al., 2015). Other studies in cognitive neuroscience focus predominantly on test-retest reliability of subject-specific results, which has been shown to be generally high in canonical GLM analyses (Brown et al., 2011; Casey et al., 1998; Friedman et al., 2007), though inter-site reliability in some cases can be low (Friedman et al., 2007).

\section{Personal experience of scientists}

How do scientists themselves perceive reproducibility and replicability? In 2016, a survey of the scientific journal Nature was answered by 1576 scientists (Baker, 2016). The survey study revealed that more than $70 \%$ of scientists failed to reproduce other scientists' work, while more than $50 \%$ failed to reproduce their own work. We assume that this refers to results 
reproduction, though this is not entirely clear from the article. One should also note that failed results reproduction might be linked to type-II errors. Around half of the respondents agreed that there is a significant reproducibility crisis, while only $3 \%$ agreed that there is no crisis $(38 \%$ agreed that there is a slight crisis). Fortunately, the study revealed that most reproducibility attempts were accepted for publication, irrespective of whether replication of results succeeded or failed. Around one-third of respondents indicated that their lab had established procedures for reproducibility, which includes peers reconducting the study.

\section{Interim conclusion}

Estimates of reproducibility of scientific results in life and social sciences are often low; but in some cases moderate or even high. The reproducibility rate depends on (see also Table 1) the scientific field (see, e.g., Wilson \& Wixted, 2018), the power of original and replication study (see, e.g., Klein et al., 2018 for the effect of sample size), the number of reproductions of a study (see our paragraph on meta-analysis across reproductions of studies), the definition of reproducibility (e.g., results or inferential reproducibility), the thresholds chosen for dichotomization of statistical evidence (see, e.g., Klein et al, 2018, though the effect was small), the statistical framework (Etz \& Vandekerckhove, 2016), and the similarity between the original and reproduced research design (e.g., same or different data). Interpretation of the results of a reproducibility study should take all these factors into account. As such, from a null-hypothesis testing (NHST) perspective, high reproducibility can be achieved through aggregation of results from multiple tests on the same hypothesis with high prior-odds using the same design. Though this is probably not the most exciting research, and may not be as novel, it is important to establish a solid knowledge foundation. A possible formalism of reproducibility has been outlined in Devezer et al. (2020), who argue that much of the 'scientific reform' literature lacks formalism and methodological rigor, and suffers from overgeneralization. In some cases, irreproducibility can tell us something about the context in which scientific findings hold.

\section{Success of a reproducibility study Reference depends on:}

Definition of reproducibility (e.g., methods, Goodman et al. (2016) results, or inferential reproducibility)

Power of original and replication study $\quad$ E.g., Klein et al (2018)

Scientific field (likely related to the base rate of See, e.g., Wilson \& Wixted, 2018 true effects)

Number of attempted reproductions and their See, e.g., Klein et al. (2014) and aggregation

Camerer et al. (2018) 
Similarity between the original and reproduced study

See, e.g., Klein et al. (2014) for a study with high similarity

The thresholds used for dichotomization of See, e.g., Klein et al. (2018) statistical evidence.

Table 1. List of important determinants of reproducibility in a broad sense. Note that this is not meant to be an exhaustive list.

\section{Questionable research practices (QRPs)}

\section{Definition and examples}

Although it is difficult to conduct scientific studies on the causes of issues with reproducibility of scientific studies, it is often argued that the following practices might have partially contributed to, or helped to maintain it (but see also Fanelli, 2018, for some counterarguments): publication pressure (based on the number of publications as well as the journal's impact factor), publication bias (e.g., because often statistically insignificant and negative results are often not published; see e.g., Schmucker et al., 2014), and low statistical power of individual studies (see, e.g., Baker, 2016; Begley \& Ellis, 2012; Button et al., 2013; Kong et al., 2019). For example, very low power implies that even if an effect is detected using a statistical test it likely reflects a non-existent effect hence leading to irreproducibility of results (Button et al., 2013) and publication of false claims (loannidis, 2005). Some of these issues have intuitively a straightforward solution: publication bias for example can be easily reduced by being open or transparent about every aspect of a study (see the paragraph on transparency and openness) and by publishing insignificant results (see the paragraph on publication of statistically insignificant effects). However, in reality this requires a lot of effort, and often acquisition of new skills and knowledge is desirable (e.g., new statistical perspectives).

Most of these presumed causes of irreproducibility are either symptoms (e.g., publication bias) or causes (e.g., publication pressure) of questionable research practices (QRPs), which are practices performed by researchers whose use is debatable, and often harmful, but cannot be categorized as blatantly fraudulent such as falsifying data (John et al., 2012). QRPs include (see also Table 2) selective reporting of dependent variables, analyses and experimental conditions, selective exclusion of outliers, hypothesizing after results are known (also known as HARKing), repeated analyses without adjusting the alpha-level, salami slicing (i.e., inappropriately dividing one coherent study in multiple articles to increase the number of publications), and reconducting analyses with different statistical tests while only reporting the analysis that led to statistically significant effects (Artino et al., 2019; Banks et al., 2016; Eva, 2017; John et al., 2012; Schoenbrodt, 2015). Many of these practices fall under the umbrella of "p-hacking", described as to "collect or select data or statistical analyses until nonsignificant results become significant" (Head et al., 2015, p. 1). QRPs have been described in colorful ways. For example, John et al. (2012) described QRPs as follows: 
"QRPs are the steroids of scientific competition, artificially enhancing performance and producing a kind of arms race in which researchers who strictly play by the rules are at a competitive disadvantage. QRPs, by nature of the very fact that they are often questionable as opposed to blatantly improper, also offer considerable latitude for rationalization and self-deception." (p. 524)

Similarly, Artino et al. (2019, p. 76) noted "[...] QRPs exploit the ethical shades of gray that color acceptable research practice [...]".

It is important to note that these QRPs do not only have an effect on knowledge accumulation, but also can have real-life health consequences, as publication bias can give medical doctors biased information about the medicine they prescribe (see, e.g., Turner et al., 2008; Chan et al., 2014).

Name of the QRP Description of the QRP

P-hacking

Changing or adding analyses for the mere reason to make an effect statistically significant.

E.g., selective exclusion of outliers

Hypothesizing after results are Changing the hypothesis post-hoc to make them in known (HARKing) line with the found results.

Salami slicing

Dividing a coherent study in multiple articles to increase the number of publications.

Selective reporting

Not reporting parts of the data (or data features), analyses, parts of the design, and results (amongst others) to make the study look more convincing.

Table 2. A list of examples of questionable research practices. Note that this list is not meant to be exhaustive but rather exemplary.

\section{Scientific research on QRPS}

In the past decade, research (i.e., meta-research; loannidis et al., 2015) has been conducted on the use and consequences of QRPs in science. In a systematic review, Banks et al. (2016) studied the use of QRPs in social and organizational sciences and included studies that used survey analyses (both self-report and observer-report), sensitivity analyses, and behavioral observations. Their final analyses included 64 studies on QRPs that fit their inclusion criteria. They found that the vast majority of included studies on QRPs show compelling evidence of the widespread use of QRPs in social and organizational sciences, including $p$-value manipulation, selective reporting of statistically significant results (as opposed to nonsignificant results), and withholding to report methodological details, amongst many others. The authors concluded as follows: 
"Yet, engagement in QRPs is occurring at rates that far surpass what should be considered acceptable. Thus, some type of action is clearly needed to improve the state of our science." (p. 328)

The authors concluded that QRPs are (more) often used when initial hypotheses are not supported.

Similarly, in a recent large-scale survey study $(N=590)$ on QRPs in health professions education, $90.3 \%$ of respondents reported to have at least once committed a QRP (Artino et al., 2019). Most of these committed QRPs were minor, including honorary authorships (61\% of respondents indicated to have done this at least once), citing articles that were not read ( $50 \%$ of respondents indicated to have done this at least once), and citing certain papers to please the editors or reviewers ( $49 \%$ of respondents indicated to have done this at least once). Though these practices can be harmful, other practices that were conducted less frequently may be more harmful, for example collecting more data after observing that the results were almost significant (14\% of respondents indicated to have done this at least once) and deciding whether or not to exclude nonoutlier data after looking at the impact on results $(12 \%$ of respondents indicated to have done this at least once). Fabricating data was reported by $2.4 \%$ of respondents. Note that this study relied on self-report measures, which could have had an influence on the results. Other studies reporting misconduct of peers show higher percentages for falsification (Fanelli, 2009, found reported $\sim 14 \%$ ). Banks et al. (2016) proposed some recommendations to discourage the use of QRPs in science. These recommendations included journals being more explicit about what practices are (not) acceptable, the use of Registered Reports (see our paragraph on preregistration), and to provide courses on the use of sound research practices.

\section{Base rate or prior odds of true effects}

Though it is intuitive to attribute issues concerning reproducibility to QRPs, some simulations suggest that the base rate of true effects might often be a more important determinant of results reproducibility (Ulrich \& Miller, 2020). Comprehensive empirical studies could disambiguate the separate effect of both QRPs and base rate.

\section{QRPs and analytical flexibility}

Analyzing a dataset comes with many choices regarding processing steps, and often there is high analytical flexibility since seldomly a golden processing standard has been described (Carp, 2012; Simmons et al., 2011). In combination with the common questionable research practice (QRP) to only publish results that are in line with one's a priori hypothesis (Chan et al., 2004; Chan et al., 2014; Jones et al., 2015), analytical flexibility might allow researchers to present compelling evidence for almost any hypothesis (Carp, 2012; Simmons et al., 2011). In basic functional MRI research for example, there are many potential software packages one can use (e.g., SPM, FSL, AFNI), with each having multiple versions and updates, and many preprocessing steps to (not) use, where each included preprocessing step increases the dimensionality (Poldrack et al., 2008; Gorgolewski \& Poldrack, 2016). When one uses advanced methods (for example dynamic causal modelling; Friston et al., 2003), even more options are available. 
Another issue with analytical flexibility arises when one aims to correct for multiple statistical comparisons, which are used when researchers perform multiple statistical tests and do not want to inflate the number of type-I errors. Multiple comparisons corrections are mainly a concern when multiple tests are conducted, and are mostly applied to control the family-wise error rate (FWER; i.e., the probability of making at least one type-I error) or to control the falsediscovery rate (FDR; i.e., the proportion of falsely rejected null-hypotheses). Often researchers have to decide what comparisons they will bundle (in other words: consider as a family) for which to control the FWER or FDR. The difficulty here lies in what to consider as a family i.e., a set of coherent tests - for which to correct for multiple comparisons. There are many opinions about what should be included in a family of tests. Some argue that a researcher should correct for all comparisons they do in their lifetime (Perneger, 1998), others say that you should only correct for multiple tests that concern the same hypothesis or theory (Lakens, 2016; Rubin, 2017). The difficulty with the former is the sharp increase in type-Il error rates, while the difficulty with the latter is the decision concerning what tests one would consider testing the same hypothesis or theory. Researchers might decide post hoc what multiple comparisons correction they will apply and what they consider as a family (whether or not testing the same hypothesis), depending on the effects they (don't) want to show. The flexibility and uncertainty in applying multiple comparisons controlling methods might unfortunately lead to biased results and unreliable research conclusions, and results of studies might actually show what the researcher wanted to show a priori - or what other researchers have shown beforehand in other studies - while there is no straightforward argument against it. The issue of multiple comparisons correction has also implications for the use of $p$-values in exploratory research, which are discussed in the paragraph on expanding our statistical toolbox. Thorough preregistered and documented statistical plans might alleviate part of the issues of post hoc choices (see our paragraph on preregistration).

\section{Cognitive biases}

Cognitive biases are mental processes that may lead to errors in reasoning and thinking (Cooper \& Meterko, 2019; Nosek et al., 2018). The confirmation bias (also referred to as hypothesis myopia; Nuzzo, 2015) is a well-known cognitive bias, which describes that individuals mainly focus on (and reinterpret) information to confirm their existing beliefs and disregard information that is not in line with their thinking (Nickerson, 1998; see https://catalogofbias.org/biases/ for a catalog of mainly cognitive biases). It has been suggested that cognitive biases could partially cause issues concerning reproducibility (Nuzzo, 2015). Scientists might for example only look at the part of the evidence that confirms their prior belief (i.e., the confirmation bias) leading to potentially biased reporting of the study. Or they might not think about alternative (likely) explanations for their results except for the one they already had in mind, which might lead to overconfidence in their theory (see also Nosek et al., 2018). Munafò et al. (2017) and Nosek et al. (2018) suggested that blinded data acquisition and analysis might be a powerful tool against cognitive biases. However, blinding might be difficult to apply in exploratory research. Other suggested solutions against the influence of cognitive biases on science include motivating scientists to consider and test alternative explanations, and transparency of research material (Nuzzo, 2015). 


\section{A note on self-correction in science}

Some might argue that self-correction in science (which means that theories, hypotheses, and claims that are not supported by sound empirical evidence will not be used further) might protect science from accepting false claims. However, self-correction in science is not selfevident; in other words, there is bias toward citing work that supports claims over work that does not support claims (Bordignon, 2020; Greenberg, 2009; loannidis, 2012). For example, Greenberg (2009) found evidence for citation biases in favour of articles that provide positive support for claims in neurobiological research on Alzheimer's Disease (in drug research a similar positive reference bias has been observed; Gotzsche, 1987). They also found evidence that citations are sometimes misused, for example by making false claims about the cited study. Bordignon (2020) found that critical citations (i.e., criticizing previous work) are uncommon, and argued for (anonymous) post-publication peer review as a promising avenue for scientific self-correction. More examples of studies on citation bias can be found in Glasziou et al. (2014). The presence of papers researching self-correction in science could in itself be interpreted as scientific self-correction.

\section{Wasted resources}

It has been estimated that the vast majority of investment in biomedical research is wasted, which has been attributed to underreporting of negative research findings (i.e., not statistically significant or in contradiction to what has been hypothesised), a focus on short-term success, and personal career ambition, amongst many others (Macleod et al., 2014).

\section{Interim conclusion}

In summary, research has shown that QRPs are often applied in scientific research. Although it is difficult to determine the extent to which issues concerning irreproducibility are caused by the use of QRPs, they most certainly add (at least some) confusion to scientific claims, and subsequently slow down scientific progress. This is a paradox of quantity-focused publishing in life and social sciences; it seems as though we are progressing at a fast pace, but because dubious practices are present, we spend time disentangling claims that are based on sound scientific evidence and those that are not. However, prior odds of a hypothesis being true should always be taken into account when interpreting reproducibility.

Luckily, solutions against QRPs have been proposed. Blinded data analysis and data acquisition might be a potential solution against some QRPs and cognitive biases (Munafò et al., 2017). A shift in culture to publishing and valuing null results (i.e., non-significant results from a NHST perspective) might lead to less publication bias in the literature. Making researchers aware of cognitive biases might also have a positive influence. Alternatively, some scientists proposed to do 'slow science', arguing that scientists need time to think, read, and prepare studies thoroughly (http://slow-science.org/; see also Alleva, 2006).

Figure 1 gives a scheme of possible paths leading to irreproducibility. 


\section{A scheme of possible paths leading to irreproducibility}

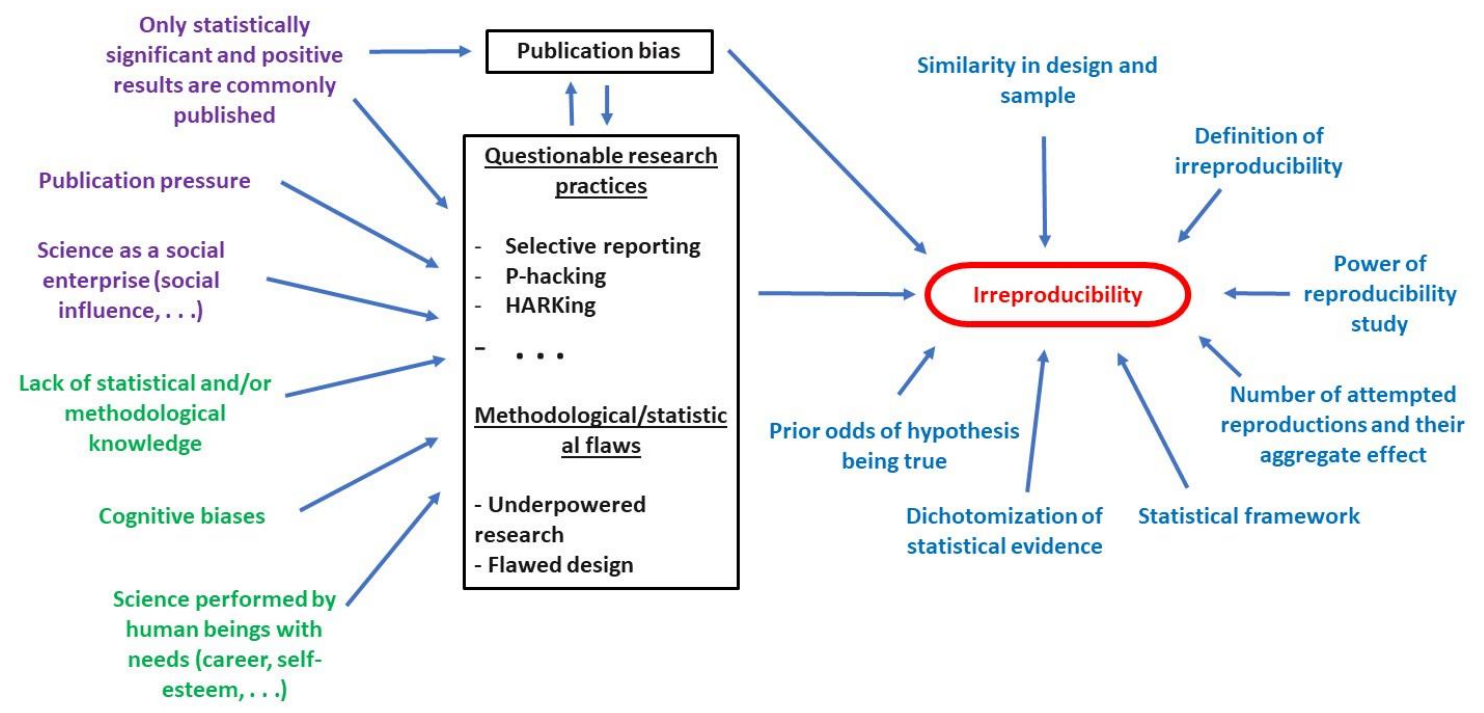

Figure 1. A scheme of possible paths leading to irreproducibility, abstracted from the literature. This scheme could be used to formalize and guide further meta-science (see e.g., Devezer et al. 2020). It is important to note that not all the effects have been studied extensively (e.g., the impact of social influence on QRPs), and that some are controversial (see, e.g., Fanelli, 2018, for counterarguments regarding the influence of QRPs on reproducibility). We divided the variables in four different categories: Individual (green), communal (purple), methodological/statistical (blue), and explanatory (black) variables. Note that some variables can be argued that some belong to multiple categories (e.g., science as a social enterprise). Also note that we also did not display possible interactions between causes (e.g., the influence of publication pressure on cognitive biases).

\section{Improving the way life and social sciences are conducted}

In the following section, we aim to present ways on how we could improve the way life and social sciences are conducted. Based on what research has shown and what researchers have suggested (e.g., Munafò et al., 2017; Chan et al., 2014), we summarize the literature in six categories of improvement, which are shown schematically in Figure 2 . We also outline software and online platforms that researchers might use to improve their research, most of which have been developed in the past decade. 


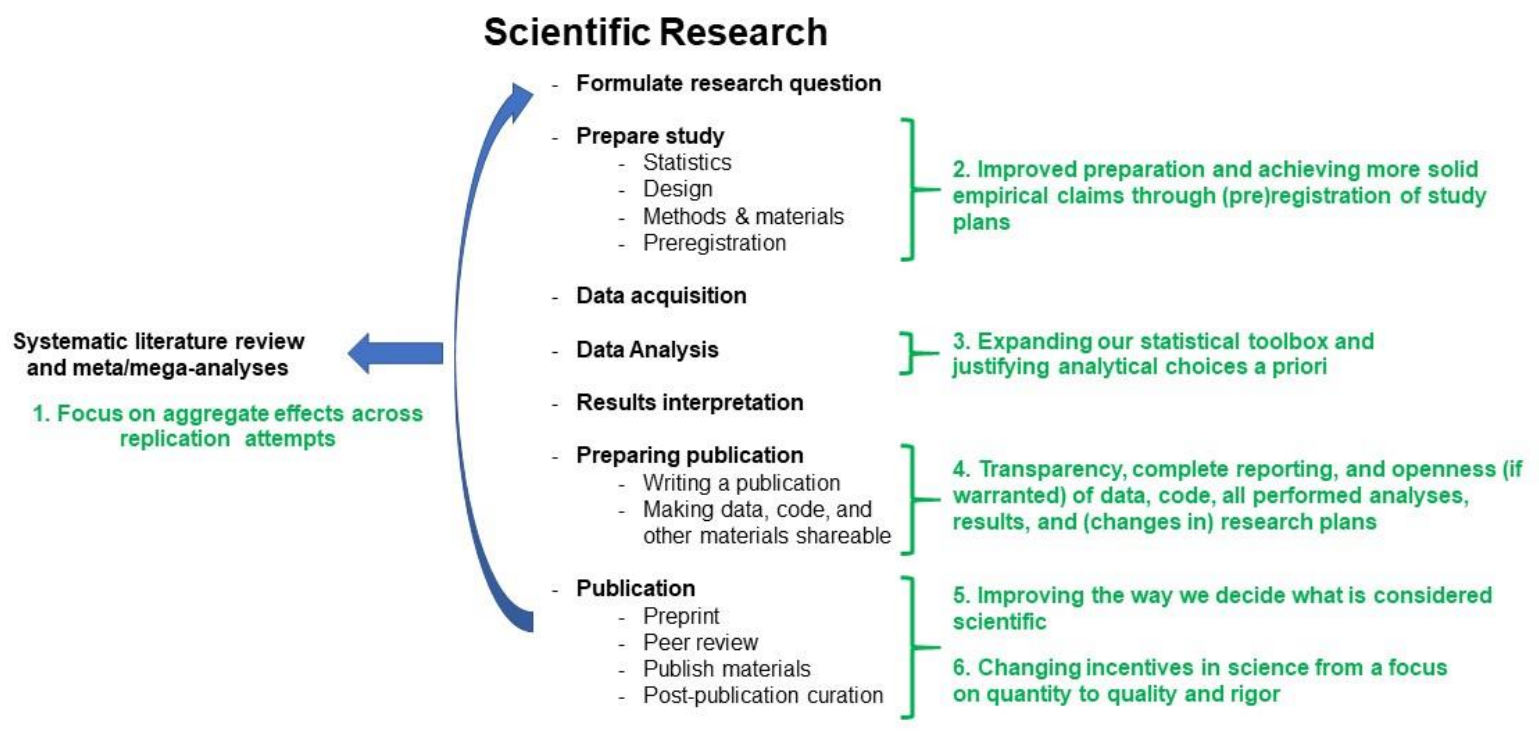

Figure 2. A schematic of improvements outlined in this review located the steps involved in scientific research.

\section{Improvement 1: Focus on aggregate effects across replication attempts}

\section{Meta-analysis across reproductions of studies}

The generally low to moderate estimates of reproducibility in life and social sciences warrants researchers from basing their conclusions on only one study. Instead, researchers should focus on aggregate effects across multiple replications of studies, which can be achieved using meta-analysis. For example, a replication study in psychology (including social and cognitive psychology studies) performed by many different labs (Klein et al., 2014) showed that most of the included effects can be reproduced successfully (referring to results reproduction) when effects are aggregated across reproducibility studies (results of $77 \%$ to $85 \%$ of studies were able to be reproduced). Moreover, aggregate effects across reproduction attempts were estimated with high precision because they were informed by many (reproducibility) studies (though these effect sizes were in some cases heavily over- or underestimated in the original studies compared to the meta-analyses). Similarly, Camerer et al. (2018) used meta-analysis to estimate effect sizes across results of the original and replication studies, which resulted in a results reproducibility of $76 \%$ (note that the original results were included in the meta-analysis). In general, Klein et al. (2014) and Camerer et al. (2018) show that reproducibility of results can be high when one aggregates results across multiple studies, and that this allows more precise estimates of effect sizes.

\section{Issues using meta-analysis and potential solutions}

\section{Issues}

Even meta-analytic studies may not provide useful information if the studies within are not conducted according to strict hypothetico-deductive scientific principles (see our paragraph on the hypothetico-deductive scheme). Meta-analyses applied to studies that have an inherent 
bias toward confirming hypotheses and/or the literature, might lead to increased beliefs in significant effects and their effect sizes (Kvarven et al., 2020). In their study, Kvarven et al. (2020) compared results found by standard meta-analyses (i.e., using standard metaanalytical studies in the literature) to results shown by pure replication studies (where selective reporting and publication biases are unlikely by construction). The number of included studies was fifteen for both types (meta-analysis and replication). They observed that $47 \%$ of (aggregated) replication studies could not reject the null-hypothesis while the standard metaanalyses found statistically significant effects in the same direction as the original study. Note that the power in most replication studies and meta-analyses was high. Moreover, effect sizes computed by standard meta-analyses across studies tended to be three times as large as effect sizes in strict replication studies. Note that this replication study concerned studies in the field of psychology; however, we do not expect that the effect would be qualitatively different in other disciplines within social and life sciences.

\section{Bias-adjustment methods as solution?}

In the same study (i.e., Kvarven et al., 2020), the authors tested whether meta-analytic techniques for bias-adjustment (i.e., adjustment for publication bias) would decrease the observed discrepancy. Two out of three techniques showed very similar results compared to standard meta-analyses and thus did not have much effect. The third method showed less false-positives compared to the two other methods, but at the same time drastically decreased power, leading to $71 \%$ false-negatives (confidence intervals were very wide; something that has also been shown in Carter et al., 2019). Note that for these results (e.g., false-positive ratio) the results after bias-correction methods were compared to results shown by the replication studies. This shows that statistical publication bias-correction methods are not able to correct for the effects of QRPs in general, which was also concluded by a recent simulation study (Carter et al., 2019).

\section{Interim conclusion}

Altogether, these studies show that meta-analyses, which are very powerful methods, should focus on well-designed studies that are free of publication bias, selective reporting, and $p$ hacking; for example, studies that have preregistered their research plan (e.g., Registered Reports; see also Nelson et al., 2018). Note that a study that is reproducible does not mean the interpretation of the results is valid.

\section{Improvement 2: Improved preparation and achieving more solid empirical claims through (pre)registration of study plans}

\section{The hypothetico-deductive scheme}

Scientific research may be improved by focusing on the essence of hypothetico-deductive science (Munafò et al., 2017; Nosek et al., 2018). It is important to note that other types of scientific research exist, such as inductive and abductive research. Interesting references for inductive research include Locke (2007) and Spector et al. (2014), and an interesting reference for abductive research is Aliseda (2006). Here we focus on hypothetico-deductive 
science, because results of both inductive and abductive science should be tested rigorously using methods used in pure hypothetico-deductive science (see e.g., Nosek et al., 2018).

The hypothetico-deductive scheme starts with (1) the generation of a hypothesis (e.g., from a theory), which is followed by (2) designing a study that allows to test the hypothesis, (3) collecting data according to the design, (4) testing the predefined hypothesis, (5) interpreting results, and (6) disseminating results (e.g., through peer reviewed publications; see also, Munafò et al., 2017). Pure adherence to this scheme would lead to strict testing of the hypothesis in question. This scheme is often not followed strictly in contemporary science. Not adhering to this scheme in purely hypothesis-driven research might reflect problematic conduct in contemporary science (see Munafò et al., 2017). These problems include lack of replication, low statistical power, p-hacking, hypothesizing after results are known (HARKing), publication bias, and lack of data sharing (see our paragraph on Questionable Research Practices).

In order to motivate scientists to adhere to the hypothetico-deductive scheme, preregistration formats and Registered Reports have been developed (see e.g., Nosek et al., 2018), which are discussed in the next paragraph.

\section{Preregistration}

Preregistration is the disclosure of a research plan (and preferably also the reasons for this research plan) before conducting a study (Nosek et al., 2018). This disclosure should ideally be identifiable (e.g., using a digital object identifier, or short: DOI) so that it can be included in the published article that reports the results following execution of that research plan (for a study using preregistration, see Esteban et al., 2017). Preregistration can be used to "define the research questions and analysis plan before observing the research outcome" (Nosek et al., 2018, p. 1), and "committing to analytic steps without advance knowledge of the research outcomes" (Nosek et al., 2018, p. 2). It also helps reviewers to focus on the soundness of designs and analyses.

One way of preregistering studies is to register the research plan on official websites, such as https://osf.io (the Open Science Framework; see panel A of Figure 3) or AsPredicted (https://aspredicted.org/). Another way is to use the Registered Reports publishing format, where the research plan of a study has been peer-reviewed before the study is conducted (see Figure 3 panel B; Chambers et al., 2014; Nosek \& Lakens, 2014). In the first stage of peer review, the quality of the design and statistical plan is assessed. In the second stage of peer review, the executed study is compared to the preregistered plan (Nosek et al., 2018). After passing through the first stage, the study is very likely to be accepted for publication (referred to as in principle acceptance) after the study has been conducted, irrespective of the (statistical significance of) results. For a list of journals that allow Registered Reports, see https://www.cos.io/our-services/registered-reports (currently, i.e., April $15^{\text {th }} 2021$, there are 288 journals in this list).

Preregistration has been shown to be beneficial, leading to decreased hypothesis-confirming results, increased reproducibility, and improved detection of selection bias, though more research is needed to confirm these results (Nosek et al., 2018). It has also been argued that preregistration will bridge the gap between contemporary scientific practice and statistical 
theory of inference (Nosek et al., 2018). Badges for preregistered studies have also been developed (see https://osf.io/tvyxz/wiki/home/ and Panel C of Figure 3) as well as preregistration formats for (computational) models (Lee et al., 2019). Platforms to register systematic reviews have also been developed (see, e.g., https://www.crd.york.ac.uk/PROSPERO/ https://systematicreviewsjournal.biomedcentral.com/).

Ideally, reproducibility studies should be preregistered. However, only a minority of journals explicitly state that they accept reproductions, while most journals do not reveal whether they would accept such studies (see e.g., Yeung, 2017 for neuroscience). It might be a good idea for journals to be more explicit on this topic.
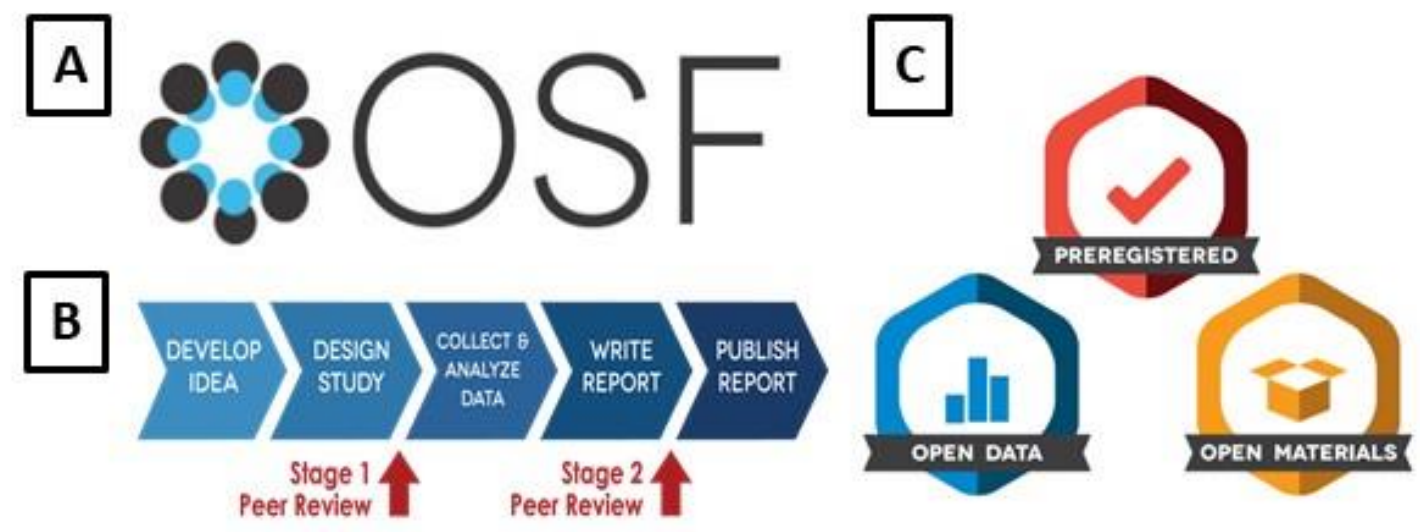

Figure 3. Some of the services and platforms provided by the Center for Open Science (COS; https://www.cos.io/). Panel A: The open science framework provides researchers with a platform to share all study-related information and to preregister studies. Panel B: The workflow for publishing a Registered Report. In summary, the research plan (or study design) is peer-reviewed before data collection and data analysis (Stage 1 Peer Review). The study is peer-reviewed a second time after the results have been written down (Stage 2 Peer Review). This time the study is mainly judged based on the adherence to the original research plan. Note that Registered Reports are now also made possible for studies that use preexisting data. Panel C: Open Science Badges which are added to the top of articles that are preregistered, share data and/or share other materials. All figures were reprinted from the Open Science Framework (n.d.), retrieved from https://cos.io, copyright CC BY 4.0, https://creativecommons.org/licenses/by/4.0/.

\section{Exploratory research and hypothetico-deductive science}

Importantly, the application of the hypothetico-deductive scheme for hypothesis-driven studies does not exclude exploratory research (Munafò et al., 2017). Exploratory research can be conducted without following this design rigorously and can generate interesting hypotheses or methods for the hypothetico-deductive method to test or use (Munafò et al., 2017). However, unfortunately a lot of the exploratory research has not been very open nor transparent. For example, publication bias (i.e., only publishing significant results) and selective reporting have precluded the publication of all results (Banks et al., 2016; John et al., 2012). Similarly, data and code are often not open (see paragraph on transparency and openness). Moreover, 
methods that are published often do not show the simulations that did not work, and why the present parameter settings were chosen. Therefore, research environments should incentivize scientists to be more transparent and open, even when conducting exploratory research, and to disseminate both good and bad aspects of studies (even mistakes or errors).

\section{Interim conclusion}

Specific platforms such as the Open Science Framework have been developed to preregister a research plan, mostly applicable when researchers aim to conduct hypothesis-driven research. Registered Reports are a formal way of reviewing a study design before conducting a study. Exploratory research is less suited for preregistration but should nonetheless be very transparent on what analyses have been conducted (and not just show the analyses that reveal pleasant results). Preregistration could also help researchers in improved planning of their study and can inform scientists whether other scientists are already studying what they aim to study, possible reducing unnecessary duplication (note that it is often useful if independent groups study the same hypothesis to check reproducibility, however, too many groups studying the same hypothesis could be regarded as wasteful; see also Macleod et al., 2014). However, one should be aware that reproducible research is not necessarily valid nor truthful (Devezer et al., 2020), as results might not reflect the underlying truth one seeks to find, and interpretation might be biased. Though, reproducibility shows whether it is worthwhile interpreting results and building theories based on observations.

\section{Improvement 3: Expanding our statistical toolbox and justifying analytical choices a priori}

The most widely used form of statistics in scientific publications is null-hypothesis significance testing (NHST). The abundant use of the $p$-value in science has received criticism (Nuzzo, 2014), and it has been argued that NHST is often used without proper justification (Szucs \& loannidis, 2017b). In recent years, a debate has been taking place on whether and how we should change the use of statistics in science. Since there is no straightforward comprehensive answer, and studies should almost be checked case-by-case on what statistics are most optimal, we aim to give an overview of the most recent debates on alternatives to the standard use of NHST and the significance level.

\section{The (recent) debate on the use of NHST and the significance level}

\section{Redefine statistical significance (Benjamin et al., 2018)}

In 2018, Daniel Benjamin and $\sim 70$ collaborators proposed to change the standard $p$-value threshold from 0.05 to 0.005 for new scientific discoveries. They argued that the current standard $p$-value threshold of 0.05 results in too many false-positives as well as decreased replicability, and that the term 'suggestive' (instead of 'significant') would be a better way to refer to evidence associated with $p$-values between 0.05 and 0.005 . Importantly, they also argue that this new threshold should only be applied for new discoveries, not for replication studies. The researchers also stated that reducing the $p$-value threshold should be conducted additionally to other changes in scientific studies, including preregistration and transparency. 
In a response, Daniel Lakens and $\sim 90$ colleagues proposed that instead of adopting a new standard value for $p$-value thresholds, researchers should be encouraged to (a priori) think about, and decide on, appropriate thresholds themselves, and to additionally focus on other metrics such as practical relevance and other aspects of research designs (e.g., effect size of interest). They argue that lowering the standard $p$-value threshold to 0.005 would lead to less resources for replication studies, more convenience sampling, and would create the risk of focusing too much on single $p$-values. Similar to Benjamin et al. (2018), they also argued for more transparency and preregistration.

\section{Abandon statistical significance (McShane et al., 2019)}

In 2019, Blakeley McShane and colleagues proposed to stop applying thresholds to $p$-values, instead treating them as continuous, to drop null-hypothesis significance testing (NHST) as default statistical paradigm in science, as well as to consider other factors such as prior evidence and proper research design with equal importance. They criticize the NHST framework by pointing to the implausibility of a strict point null-hypothesis (e.g., the population parameter is equal to zero) and zero systematic noise (e.g., no systematic sampling error). They also argue that it makes no sense to categorize evidence, and raise concerns that $p$ values are a poor measure of evidence in favour of (or against) a hypothesis (since $p$-values are based on conditioning on the null-hypothesis). The proposal to retire statistical significance has been signed by more than 854 scientists from 52 countries in Amrhein et al. (2019). Similar concerns have been raised by Cumming (2014), who also advocates for the use of effect sizes, confidence intervals and meta-analyses, and recommends not to report $p$-values nor even mention NHST.

\section{Random as opposed to fixed effects (Yarkoni, 2019b)}

Others such as Yarkoni (2019b) have argued for improving the generalizability of effects by using random effects models (as opposed to fixed effects models). By modelling different sources of variance, random effects models enable to generalize findings beyond the specific materials used in studies (e.g., specific set of stimuli). This would allow closer compatibility of theories (and their corresponding hypotheses) with statistical inference. Additionally, he argued for more conservative conclusions in scientific papers, increased focus on practical utility of research, and to make predictions that better discriminate between different theories (which often are riskier predictions). Note that Yarkoni (2019b) specifically focused on psychological science; however, the same arguments can be made for other disciplines within life and social sciences as well.

\section{Alpha-level versus statistical power}

Though some researchers have argued for more conservative alpha-levels, research has also shown that power is low in some scientific disciplines such as psychology and cognitive neuroscience (Poldrack et al., 2017; Szucs and loannidis, 2017a). In studies with very low statistical power, it is not unlikely that statistically significant results reflect null-effects (Button et al., 2013). Therefore, when using conservative alpha-level thresholds, higher sample sizes are advised to maintain adequate statistical power.

Similarly, Fiedler et al. (2012) argued for a focus on false-negatives over false-positives, and other authors have even argued that selective publication of positive results can be effective 
(e.g., de Winter \& Happee, 2013). However, such conclusions often depend on scientific selfcorrection being true, which is not necessarily the case (see our paragraph on self-correction in science).

\section{Publication of statistically insignificant results}

Statistically insignificant effects are not useless nor uninteresting, but sadly they are often not published (see e.g., Schmucker et al., 2014). Whether or not an effect or association is significant depends on many factors, including statistical power. Researchers who aim to test insignificant effects can use equivalence testing (Lakens, Scheel, \& Isager, 2018) or Bayesian statistics (see e.g., Wagenmakers, 2007). Specific collections in journals have been developed to publish null or negative results (see e.g., https://collections.plos.org/collection/missingpieces/).

\section{P-values and exploratory research}

Another question concerning $p$-values is whether they are suitable to use in non-hypothesisdriven research. Some people advise against the use of $p$-values in exploratory research (Nosek \& Lakens, 2014; Forstmeier et al., 2017). One reason for this argument is that it is usually not known how many hypotheses have been tested, which leads to an unknown inflation of the type-I error rate (Nosek \& Lakens, 2014; Forstmeier et al., 2017). Another reason for this argument is that there is no explicit null-hypothesis (nor an alternative hypothesis) in exploratory research (Neuroskeptic, 2015). It has also been argued that $p$ values can be continued to be used in exploratory research, as long as we make a clear distinction between $p$-values used in exploratory research and $p$-values used in confirmatory research (preferably giving the latter a different name to make a clear distinction; Neuroskeptic, 2015).

Others have argued that some of the above arguments only hold when the family of hypotheses for which to do multiple corrections is assumed to include different null-hypotheses (Rubin, 2017). For example, let us say a scientist conducts two tests: one test for the relation between phenomenon $\mathrm{A}$ and $\mathrm{B}$, and another test for the relation between phenomenon $\mathrm{C}$ and D. The null-hypotheses concern different phenomena, and according to Rubin (2017) no correction for multiple comparisons should be done. Rubin (2017) also argued that even in the case of different tests involving different hypotheses, the applicability of $p$-values to exploratory research depends on the family of hypotheses you want to correct for. For example, it would be possible to use $p$-values in exploratory research as long as the definition of the family of hypotheses for which to correct for is reasonable in size (and all conducted tests within this family are taken into account). In other words, if one would define the family of hypotheses for which to correct statistically as reasonably small, or if one would wish to correct for incorrect rejections of the same null-hypothesis, then one can safely use $p$-values in exploratory research, at least according to Rubin (2017).

\section{Bayesian statistics and other alternatives}

Some researchers have proposed Bayesian statistics as an alternative and/or addition to frequentist NHST (see, e.g., Quintana \& Williams, 2018; Wagenmakers, 2007; Benjamin et al., 2018). Bayesian statistics can be used to quantify evidence in favor of, or against, the null- 
hypothesis relative to alternative hypotheses. Another alternative to NHST is statistical learning (see, e.g., Bzdok, 2017).

\section{Statistics and scientific theories}

Some researchers have argued for better theorizing and use of theories in science (Fiedler, 2017; Muthukrishna \& Henrich, 2019). Fiedler (2017) advised to formulate sharp hypotheses based on strong theories that are difficult to predict from other theories (something that has also been suggested by Yarkoni, 2019b). Muthukrishna \& Henrich (2019) similarly advocated for the development of theories and theoretical frameworks in psychology, which can be used to formulate clear hypotheses and predictions.

\section{In perspective and interim conclusion}

In their article concerning research practices in psychology, Nelson et al. (2018) argued that the debate concerning $p$-values is not novel and concluded that actually any type of statistics can be problematic if they are interpreted incautiously. Although this doesn't mean we shouldn't think about improvements to the common use of null-hypothesis significance testing, as well as the use of alternatives, it warns us about blindly using any statistical method. $P$ values are an interesting addition to effect sizes and respective confidence intervals (and in specific cases clinical relevance), but $p$-values should not be used to decide whether or not a study gets accepted for publication, nor do they necessarily directly reflect whether an effect is practically relevant. This practice leads to a huge publication bias, selective reporting, and hence is harmful for science, as has also been outlined in our previous paragraphs.

$P$-values are also often used in contexts in which they shouldn't be used (e.g., in the absence of a clear hypothesis; Szucs and loannidis, 2017b) and often lead to overly strong conclusions. If one opts to apply NHST, it might be most advisable to think beforehand about applicable thresholds for $p$-values and power (Lakens et al., 2018), which will depend on the specific study. On the other hand, effect sizes (with corresponding confidence intervals), equivalence testing (see, e.g., Lakens, Scheel, \& Isager, 2018), Bayesian statistics, and machine learning can be used alternatively, or additionally, to (the standard use of) $p$-values. Munafò et al (2017) suggested that improved statistical and methodological training could help bolster the quality of scientific research.

We have summarized suggested improvements to statistics in social and life sciences in Table 3. 
Justify statistical choices (e.g., alpha level, E.g., Lakens et al. (2018) statistical model) a priori

Find a balance between alpha-level and power

See, e.g., Lakens et al. (2018)

Publish null-results (or very small effects), because they are useful and can be tested statistically using equivalence testing and

See, e.g., Lakens, Scheel, \& Isager (2018)

Bayesian statistics

Improve statistics by including methods outside classical frequentist statistics, for example

See, e.g., Wagenmakers

Benjamin et al. (2018)

statistical learning and Bayesian statistics.

Use and develop theories to formulate sharp hypotheses (with reasonable prior odds) or See, e.g., Fiedler (2017) predictions that are unlikely to be in line with other theories

Table 3. List of suggested improvements to the use of statistics in the social and life sciences.

\section{Improvement 4: Transparency, complete reporting, and openness (if warranted) of data, code, all performed analyses, results, and (changes in) research plans}

To ensure that research can be reproduced, quality checked, and efficiently replicated, researchers should be as open and transparent as possible. Transparency and openness are related but different concepts. Transparency has been denoted as "[...] the clear, accurate, and complete reporting of all components of scientific research." (The Society for Research in Child Development, 2019, p. 2). This concept includes the disclosure of subject characteristics, software and hardware details and versions, and complete reporting of methodology, amongst others. Transparency also includes stating specifically under what conditions data and code will be shared. Openness, on the other hand, has been referred to as "[...] sharing of scientific resources, such as methods, measures, and data, in order to further scientific advances." (The Society for Research in Child Development, 2019, p. 2). Openness includes sharing of materials used in scientific studies, such as data and code without costs or obligations. Both transparency and openness are important in science, since they allow other scientists to form a well-informed opinion about the study in question, and both foster reproducibility and replicability. Sharing of research material (e.g., data sharing after being contacted by other researchers) has unfortunately not been found to be successful in the past decades. Research has shown that in studies on genetics and psychology, only (approximately) $26 \%$ to $53 \%$ of authors were able to (and were willing to) provide the complete data used in their studies (Campbell et al., 2002; Magee et al., 2014; Wicherts et al., 2006). 
Similarly, sharing of code (required for e.g., statistical analyses) has been supported, but has also been shown to be suboptimal (Eglen et al., 2017; Stodden et al., 2018). Similarly, research reporting (e.g., methods) has shown to be poor (it has been estimated that at least $50 \%$ of research reports are unusable because of incompleteness and/or low quality; Glasziou et al., 2014). Though precise estimates of the percentage of scientists sharing research materials are difficult to make and will likely depend on the scientific discipline.

Fortunately, policies have been changing and an increasing number of journals and publishers require authors to make software and code available (see, e.g., Gewin, 2016; Hanson et al., 2011). Availability of both code and data is likely to foster reproduction and replication, as well as encouraging bug-checking, further code development, and reuse of data by other studies, which together save resources including taxes and students' university admission fees. Domain-general software platforms such as GitHub (https://github.com), GitLab (https://gitlab.com/), and Code Ocean (https://codeocean.com) have been used to share code for analyses of scientific data, and to disseminate open-source software packages. These platforms also allow scientists to collaborate on software projects and to quality check each other's code.

In neuroscience specifically, platforms such as OpenNeuro (https://openneuro.org/) have been developed to openly share neuroimaging datasets, and the 'Brain Imaging Data Structure' (BIDS; https://bids.neuroimaging.io/; Gorgolewski et al., 2016) has been developed to homogenize the structure of datasets, which fosters time-efficient use of datasets and development of code tailored to this format (see, e.g., fMRIPrep, Esteban et al., 2019; and MRIQC, Esteban et al., 2017), allowing the same code to be easily applied to multiple datasets. Note that similar homogeneous standards for the structure of computational models have also been proposed (Poldrack et al., 2019). Platforms such as the "Neurolmaging Tools \& Resources Collaboratory" (NITRC; https://www.nitrc.org/) are used for data as well as software sharing. Practical guidelines and advice for code and data sharing have been outlined in (amongst others) Gorgolewski and Poldrack (2016), and Eglen et al. (2017).

Fairness of co-authorship when using shared data and code (whether or not completely open) can be a subject of discussion (see, e.g., Refining our policies on co-authorship requirements, 2017). One could for example argue whether or not the researchers involved in data acquisition should be co-author(s) of all subsequent studies using the data. Moreover, data sharers should allow publication of results that might contradict the original study's results. An ethical consensus should be reached beforehand when using shared materials. One option is to use Creative Commons licenses (https://creativecommons.org/).

Making data completely open can sometimes be unwarranted, especially when sensitive data is shared. However, in order to allow other researchers to reuse and re-analyze such data, it should still be findable and (conditionally) accessible, for example through (fair) access forms and requests. Guiding principles for sharing scientific data have been outlined in Wilkinson et al. (2016). In a nutshell, the article proposes four principles of data storage and data sharing, summarized with the acronym FAIR: Findability (e.g., data are findable through internet searches), Accessibility (e.g., existence of an open protocol to access the data), Interoperability (e.g., use of broadly applicable language for knowledge representation), and Reusability (e.g., a clear data usage license). Importantly, FAIR principles have been endorsed by the European Commission (see Directorate-General for Research and Innovation 
(European Commission), 2018). When reusing data, one should also be aware of the risk of inflation of type-I error rate in specific cases (Thompson et al., 2020), therefore it should be clearly mentioned in manuscripts (especially when data is not openly shared) what studies have used the same data. Openly sharing data and code, as well as their documentation, likely increases quality checking by peers.

Though sharing data and code is very important, proper and clear documentation about the structure and usage of data and code is also important (Gorgolewski \& Poldrack, 2016). For example, often choices for certain settings in software code are made, but no reasons are provided, which leaves the user to find out why these choices were made, either by trying to find support for the choices in the literature or by contacting the authors of the code directly. Of course, not every analysis choice is made rationally, but it should be clear where unmotivated choices were made (often they can lead to interesting research themselves). Trying to find out why certain choices were made can soak up a lot of time, especially when the original researchers have to be contacted, which could be avoided through thorough documentation. Though, one should be aware that no documentation will answer all questions readers and users have, and that incomplete documentation should not prevent people from sharing data and code, as it is more important to share code even if it is not documented well (Gorgolewski \& Poldrack, 2016). Interestingly, a study from 2016 has shown that software bugs can stay unnoticed and uncorrected for a long time (in this case 15 years) even when source code is openly shared, hence influencing decades of neuroimaging research (Eklund et al., 2016). However, software bugs as well as messy and poorly documented code are not just an issue in scientific code and software; they have been argued to be often present, even in professional computer software and code used for climate science (Barnes, 2010).

Sharing code might be a source of stress, since it makes researchers vulnerable to other people potentially detecting bugs (similar concerns have also been raised in Gewin, 2016). However, scientific progress would likely be boosted if researchers would be able to quality check each other's code and develop it further through open and transparent collaborations.

\section{The downsides of openly sharing data and code, and how to deal with them}

One downside of openly shared data is that one does often not know (or realize) the circumstances in which the data were acquired. For example, one does typically not know to what extent protocols for data acquisition (e.g., instructions) were followed strictly. Neither does one typically know who acquired the data, and under what circumstances the data were acquired. Data acquired by a qualified professional (e.g., psychometrician, laboratory technician, medical imaging technician) compared to a student (e.g., Bachelor's, Master's, PhD) may not be of equal quality. Similarly, data acquired under time pressure could have different characteristics compared to data acquired under less time-pressure. Sometimes poor data acquisition circumstances can be identified by looking at the quality of the data. However, in many cases one cannot identify poor data acquisition circumstances, for instance when protocols for subject instructions were not followed, or when subjects felt uncomfortable during data acquisition.

Similarly, though openly sharing code is advised, bugs in openly shared code can also be overlooked and copied to code used in new studies, and in that sense, these bugs could propagate to multiple studies. However, the benefits of open code, as opposed to closed code, 
is that the error can be traced back to the original study and that the studies using the code can be detected. Though this only works if studies that use open code, openly refer to the code they used from other studies. In summary, blindly using open code and/or data is not warranted, quality checking and (in)formal contact with primary researchers often remains necessary (see also Chan et al, 2014).

In scientific areas such as data science, reviewing of code has been suggested to be beneficial (Day, 2020), which likely also extends to all areas of social and life sciences where software code is used.

\section{Sharing changes to analysis plans and all performed analyses}

Full reporting of all analyses and modification to research plans has been proposed in multiple papers to combat selective reporting (see e.g., Chan et al., 2014; Nosek et al., 2018). Not only is it important to publish code, data, changes to research plans and all performed analyses, it is equally important to publish all high-quality studies even when showing null or negative (i.e., not corroborating the hypothesis) results. Chan et al. (2014) argued that a lot of resources in biomedical research are wasted because high quality research is often not published when it shows null or negative results (e.g., showing that the opposite of the hypothesis is true). Moreover, results of clinical trials are not always published, leading to false confidence in medication (Turner et al., 2008). These publication biases could be avoided by publishing all high-quality research, and to make sure that all research is high quality. In medicine, a campaign has been launched to improve disclosure of results and methods of clinical trials (https://www.alltrials.net/).

\section{Incentives to share research material}

Unfortunately, there are few explicit incentives for sharing code as well as for sharing data (see also Gorgolewski \& Poldrack, 2016; van Panhuis et al., 2014, Friesike \& Schildhauer, 2015). However, research has shown that scientific articles that provide data are cited more often (Piwowar et al., 2007). Moreover, some agencies and journals require the data to be published (Gorgolewski \& Poldrack, 2016). Other incentives that have been shown to be effective are open data and open materials badges (see Figure 1 panel C), which are added to the top of scientific articles that have made their data or materials available (Kidwell et al., 2016; Rowhani-Farid et al., 2017; https://osf.io/tvyxz/). Such badges are offered currently (April 15 $5^{\text {th }}, 2021$ ) by more than 75 journals (Center for Open Science, n. d.), and have been shown to be effective (Kidwell et al., 2016; Munafò et al., 2017). For the full list of participating journals, see https://www.cos.io/our-services/badges. Though badges and other incentives work to some extent, they might not be rewarding enough to do all the extra work that data (and code) sharing requires. Therefore, alternatively, authors could opt to publish the dataset in the form of a proper scientific article, called a 'data paper' (Breeze et al., 2012; Gorgolewski et al., 2013), for which specific journals have been developed (e.g., Scientific Data, https://www.nature.com/sdata/). Although other journals have also published data papers (see, e.g., Filevich et al., 2017).

An excellent platform to link all parts of a study, and that allows preregistration, is the Open Science Framework (OSF; https://osf.io), developed by the Center for Open Science (https://www.cos.io). It is a free online tool that allows and promotes sharing and managing 
scientific projects, workflows, and extra information on studies (Foster \& Deardorff, 2017). It allows to link all aspects of a research project (data, code, extra material, preprint, published article, and figures, amongst others) to one centralized location (i.e., the dashboard of the scientist's OSF account), and to collaborate on projects. It also has the option to assign a digital object identifier (DOI) and copy-right licenses to projects, and to preregister projects directly (Foster \& Deardorff, 2017).

\section{A note on the term 'open science'}

Although improved ways of conducting science are often referred to as 'open science', this might not be the best term. Openness should be a means to achieve high-quality science. However, there is a danger that openness will become a goal in itself (because it might start living a life on its own), rather than a means to a goal (which is high quality science). For example, highly socially sensitive results (e.g., concerning IQ or political attitude) should perhaps not necessarily be open because scientists might fear retaliation, neither should highly sensitive personal data since deidentified and anonymous data might be reidentifiable (see, e.g., Narayanan \& Shmatikov, 2008; de Montjoye et al., 2015). Similarly, open preprint servers might be used by people with political and/or financial motives.

It has also been argued that the use of the term 'open science' is ambiguous, since it is an umbrella term for many beliefs, practices, and research foci (Yarkoni, 2019a). Therefore, it has been proposed to avoid the term open science as much as possible, and instead use more specific words to clarify what aspect of open science one points to (Yarkoni, 2019a). Therefore, a general term for improved ways of conducting science could be coined as 'transparent science', 'modern science' or '21 st century science', though other (possibly better) names might arise in the upcoming years.

\section{Interim conclusion}

Transparency and complete reporting of all aspects of a study - not just the aspects that fit our expectations and desires - are important for scientific progress. They allow others to quality check our data and code, and foster reproducibility. Incentives for sharing research material (e.g., data and code) have increased over the years (e.g., platforms have been developed, journals have changed policies). In some cases, complete openness of data might be unwarranted, since it cannot be guaranteed that highly sensitive personal data will never be re-identified. However, this might be used as an excuse for not sharing data. Therefore, researchers should be specific about why data cannot be openly shared, and about under what precise conditions the data will be shared (which is part of transparency) for which a vague 'data is available upon request'-statement is not sufficient.

\section{Improvement 5: Improving the way we decide what is considered scientific}

In order to be published in scientific journals, studies are peer reviewed (i.e., read and commented by other scientists). Scientists submit scientific articles to a journal and try to address comments in order to get their work accepted for publication in the journal. Though the present peer review system likely often improves the quality of studies, important aspects of studies are still overlooked, and peer reviews do not always have high quality (Arns, 2014). 
Peer reviews are also increasingly declined by reviewers (presumably because of 'reviewer fatigue'; Vesper, 2018), sometimes include unwanted biases (Goldbeck-Wood, 1999), and have been argued to be slow and inefficient (Yarkoni, 2012). Moreover, inter-reviewer reliability has been shown to be low, which means that a study might be accepted by a journal at timepoint A but not accepted at timepoint B (Bornmann et al., 2010). Improved peer review might be achieved by giving authors and reviewers checklists and clear guidelines (Nosek et al., 2012; Munafò et al., 2017), and by focusing on open and post-publication peer reviews as well as their evaluation (Yarkoni, 2012).

\section{Open, graded, pre- and post-publication peer reviews}

Specific online platforms have been developed for post-publication public peer reviews, such as PubPeer (https://pubpeer.com; see also Munafò et al., 2017; Yarkoni, 2012). Note that unfortunately PubMed Commons, a similar platform, has been discontinued because of low number of posted comments (see, https://ncbiinsights.ncbi.nlm.nih.gov/2018/02/01/pubmedcommons-to-be-discontinued/). These platforms might help in openly discussing papers, reporting errors that were missed by the original reviewers, and giving more feedback to the authors on their work. They can also help in commenting on, and helping to improve, publications that are not yet sent to a journal. For example, articles on preprint servers such as bioRxiv (https://www.biorxiv.org/), which is often used for research related to biology and life sciences, and PsyArXiv (https://psyarxiv.com/), which is often used for psychological research (see also https://osf.io/preprints/metaarxiv/ for research on transparency and reproducibility). However, journals could also publish their peer reviews themselves, which has already been implemented by some journals in different ways (e.g., eLife, PLoS, and F1000Research). A recent study showed that publishing reviews does not compromise quality nor acceptance rates; however, most reviewers do not want to publicize their identity together with these reviews (Bravo et al., 2019). Signing peer reviews might give researchers credit for these time-intensive tasks (Stern \& O'Shea, 2019).

Some authors (e.g., Nosek \& Bar-Anan, 2012; Yarkoni, 2012) additionally proposed to evaluate scientific articles in a graded way, either in addition to, or instead of, a dichotomous decision (e.g., publication versus no publication). This would give other scientists a more informative view on the quality of papers-at least in the eyes of peers.

\section{Alternatives to the current publication system and peer-review system}

The current publication system has been the same for a long time and has been argued to be outdated (Stern \& O'Shea, 2019; Tracz \& Lawrence, 2016). In the recent decade, new ideas have been proposed to improve the peer review and publication-system. In 2012, Yarkoni argued that many of the problems apparent in the present peer review and publication system could have already been solved by social web applications, such as public rating of reviews or comments. He proposed a post-publication peer review system that includes open scoring and ranking of studies and reviews/comments. Nosek and Bar-Anan (2012) proposed a graded peer review system, in which the article is submitted to a review system and is graded based on its quality. Subsequent revisions could then increase the grades of the article, while also ensuring version-control of the article. It differs from the current publication system, in the sense that researchers now often aim for journals with high-impact factor and submit to lowerimpact journals along the way (i.e., if they get rejected; Nosek \& Bar-Anan, 2012), which might 
lead to errors and demotivation. In 2019, Stern and O'Shea argued for a shift to an authordriven publication process, in which the author decides when a study is ready for publication. In their view, curation should happen after, instead of before, publication. More recently, Mambrini et al. (2020) developed a framework (PRINCIPIA) based on free market principles, that allows more flexible creation and development of journals, and aims to restore the balance between publishers, reviewers, and authors (e.g., reviewers would receive part of the submission fee). Reviewing of software code (see, e.g., Day, 2020) accompanying articles could be a good addition to reviewing written manuscripts.

\section{Existing implementations by journals}

Some existing journals have implemented some of these policies. Many of these journals support a more author-driven approach to scientific publications. F1000Research is a journal with a wide scope (from engineering to humanities) that publishes articles almost directly upon submission. The article is then peer reviewed, and reviews are published together with the author's names. Once the article is approved by two reviewers it is indexed in various databases (article referencing includes the review approval statuses). F1000Research has been selected as an open access publishing platform for Horizon 2020 and Horizon Europe beneficiaries (Lawrence, 2020). ELife is an open-access journal, mainly for articles in life sciences, that publishes decision letters and author's responses alongside the respective peer-reviewed articles. Recently, eLife also started publicly reviewing preprints published in bioRxiv, and has developed Executable Research Articles that allow researchers to add online executable code (similar to literate programming) to publications to increase transparency and reproducibility (Welcome to a New ERA of Reproducible Publishing, 2020). In PLOS Biology authors decide whether or not peer reviews of their articles are published, and reviewers can decide whether or not they sign their reviews. Note that this list of progressive implementations by journals is meant as an example but not meant to be exhaustive. Journal policy (e.g., progressive versus conservative) could be a factor to choose for one or the other journal.

\section{A new role for journals?}

In the recent decade, new roles have been proposed for scientific journals. Nosek \& Bar-Anan (2012) proposed that journals could (or should) promote scientific articles instead of reviewing them. In this way, peer review and promotion are separated from each other. Other authors suggest that (classical) journals could (or even should) become superfluous because they are replaced by citation databases (see, Tracz \& Lawrence, 2016).

\section{A note on preprints}

Preprints offer a way of openly sharing scientific studies with the world (for free), allow prepublication feedback (Munafò et al., 2017), and have been associated with increased citations of the consequent article (Fu \& Hughey, 2019). However, they also increase the risk of sharing low-quality studies (see, e.g., Glasziou et al., 2020, and see Besançon et al., 2020, regarding preprints on COVID-19), as well as studies that are heavily influenced by political ideology or financial interests. It might be interesting to build an online platform for preprints that are mainly accessible by scientists (to allow pre-publication peer reviews) without sharing them with the world. This would allow for wider post-publication reviews by peers other than the formal peer reviewers assigned by journals. It has also been suggested that preprints' credibility could be 
improved by adding open science content (e.g., link to data, pre-analysis plan, and other materials), and independent reproduction by peers (Soderberg et al., 2020).

\section{Interim conclusion}

Peer reviewing often improves the quality of studies, but shows problems (e.g., low reliability). To improve the peer review system, platforms have been developed for pre- and postpublication open peer review (e.g., pubpeer.com), and some journals have started making peer reviewing public. In order to lower the barrier to publish peer reviews, it is important that open peer reviewers can remain anonymous if they prefer to (though signing peer reviews gives researchers acknowledgement of their work). Preprints are a great way to allow for open pre-publication peer-review, as well as early dissemination of results, but create the risk that low-quality studies and that studies heavily influenced by conflicts of interests are disseminated (see e.g., Besançon et al., 2020).

We have summarized the literature on improving peer review in Figure 4. In summary, there is a need for a gate-keeper of quality, but this process should be open and transparent. Such transparency opens up the black box of contemporary peer review and allows to study peer reviewing in an informed and scientific way. Post-publication reviews, grading, and potential updates to the paper should become the norm after the paper has past the gate-keeping stage. Scientists can already start supporting the improved publication process by online peer reviewing of articles (pre and post-publication) using existing platforms (e.g., pubpeer), and selecting journals that openly share peer reviews (e.g., F1000Research, eLife).

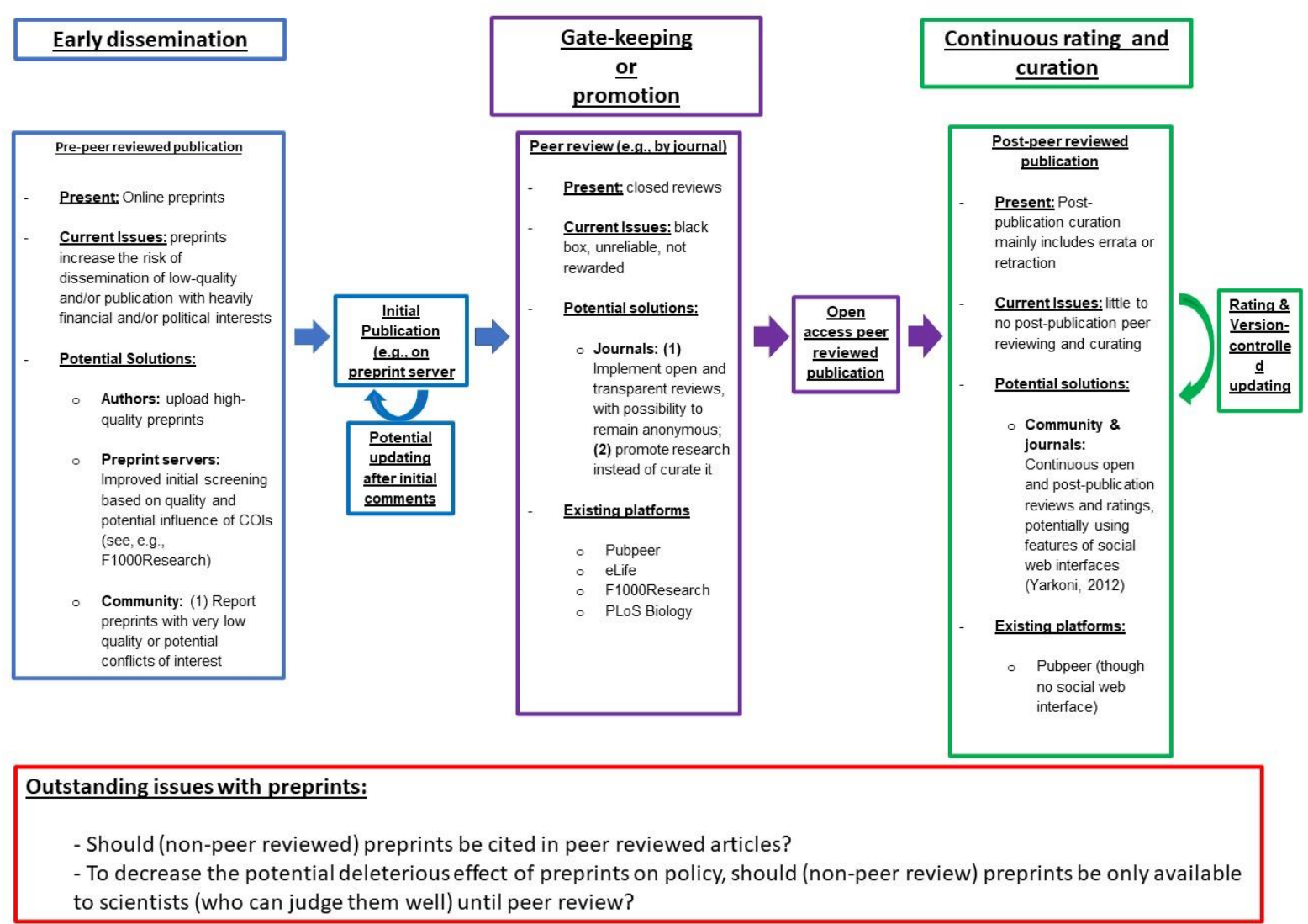

Figure 4. Summary of the literature on improving scientific peer review and the publication system. 


\section{Improvement 6: Changing incentives in science from a focus on quantity to quality and rigor}

In the second part of their series called 'scientific utopia', Nosek et al. (2012) argued for changes in incentives to improve the truthfulness of scientific claims. They argued that there is too much focus (for individuals, organizations, and institutes) on the number of publications (see also Altman, 1994), hence scientists are motivated to publish as much as possible, which could lead to 'salami slicing' coherent studies, publishing low quality and underpowered studies, and underreporting insignificant effects, among others (see our paragraph on questionable research practices). Similar concerns have been raised more recently by Schoenbrodt (2018).

The incentives for getting published - sometimes referred to as 'publication pressure' - might actually disconnect "what is good for scientists" from "what is good for science" (Nosek et al., 2012 , p. 616). In other words, it is in the scientist's interest to not present insignificant results, to not present results that conflict, and to cut a coherent study in multiple pieces to publish its parts in different articles (i.e., salami slicing), amongst others. Tijdink et al. (2014) found that $35 \%$ of 315 scientists working in medicine (Flanders, Belgium) indicated that their scientific output would be of higher quality without publication pressure, and $61 \%$ agreed that the publication pressure leads to doubts about scientific results. These results show that not all scientists (at least in medicine) consider publication pressure as problematic, though a considerable amount agrees that it causes issues. Note that Tijdink et al. (2014) found an association between scientific misconduct and publication pressure, which is in line with the results of Fanelli (2010) showing a link between publication pressure and positivity bias (i.e., publishing positive as opposed to negative support for a hypothesis), but it is hard to tell what the exact causal mechanism is. Note that there is also some counter-evidence, showing that researchers in countries with high publication pressure are less likely to be authors of retracted papers (Fanelli et al., 2015. In general it is difficult to operationalize scientific misconduct.

It is arguable whether the number of publications is a good reflection of a researcher's competence or thoroughness of conducting science, since publications are commonly only accepted if they show statistically significant effects. Finding statistically significant effects has little to do with the researcher's pure scientific competence, nor does 'getting published'. Increased competition concerning the quantity of output (now mostly focused on scientific peer-reviewed publications) eventually might lead to lower quality of papers and increased questionable research practices used to get published (e.g., to present a nice coherent story; see, e.g., Manufò et al., 2017).

However, little research has been conducted concerning the benefits for researchers if they adhere to open, transparent and modern scientific principles, and care less about the number of publications and citations. In his blog, Yarkoni (2018) argued that most of the claims concerning negative effects of not adhering to contemporary scientific incentives (e.g., openness) are not supported by data. He also argued that contemporary incentives in science are often used as an excuse not to change one's behavior. A recent survey study actually revealed slightly comforting results regarding the relation between quantitative metrics in 
science and successful academic job applications (Fernandes et al., 2020). They found that the number of first author papers and $\mathrm{h}$-index (dichotomized in an above-median and belowmedian group) did not increase the probability of a job offer. However, being a first author of a paper in journals such as Science, Nature, or Cell, as well as the total number of citations, had a positive impact on the probability of receiving a job offer. An earlier study on successfully becoming a principal investigator $(\mathrm{PI})$, showed that the number of publications and impact factor did have an important effect on becoming a PI (van Dijk et al., 2014). It is possible that the importance of contemporary scientific metrics is different for Pls versus academics in general. Considerable differences between countries might also exist and would be interesting to study in future research.

Nosek et al. (2012) outlined possible changes in incentives and scientific practices that might lead to better science. One of their suggestions was to implement checklists that authors, reviewers, and editors can use to report and judge scientific studies. Actively enforcing reporting guidelines has been shown to improve reporting of studies (Glasziou et al., 2014). In neuroscience, reporting standards (and checklists) have been developed by the Committee on Best Practices in Data Analysis and Sharing of OHBM (COBIDAS; Nichols et al., 2017; see, e.g., https://cobidas-checklist.herokuapp.com/). For systematic reviews, as well as for studies of diagnostic accuracy, reporting guidelines have also been developed (e.g., PRISMA; Moher et al., 2009; and STARD; Bossuyt et al., 2003, respectively). For more existing reporting guidelines, see Glasziou et al. (2014). Other suggestions to improve incentives included crowdsourcing replication efforts, lowering and removing the barriers to publish, and (evidently) focus on the soundness (over impact) of research, as well as opening data, workflows, and materials.

\section{Scientific metrics}

Metrics that are often used in science are impact factors (IFs) and $h$-indices, which are based on the quantity of citations and publications. Though many scientists value these metrics, they do not directly reflect scientific skills nor scientific quality (see also, Curry, 2018). For example, getting cited might be as well a consequence of being able to create a nice story, or having well-known co-authors. Likewise, having scientific impact does not imply that the impact is beneficial. Moreover, the focus on a journal's impact factor might distract people from the quality of the content of the specific scientific article and the righteousness of the journal policy. Also, the relationship between impact factor and rating of quality has been shown to be low to non-existent (Yarkoni, 2012).

In their series on 'scientific utopia', Nosek \& Bar-Anan (2012) colorfully described contemporary focus on impact factors:

"Every year that goes by with journal IFs as criteria to judge scientists is another year in the dark ages of scientific evaluation." (p. 235)

Similarly, the San Francisco Declaration on Research Assessment advised against the use of journal-based metrics like the IF to evaluate the quality of individual papers (https://sfdora.org/). The relation between current scientific metrics (impact factors and hindices) and quality of science should be studied more closely, as well as their effect on researchers' behavior. 


\section{Alternatives to the Impact Factor}

Recently, alternative (or additional) metrics have been proposed that might make scientifically more sense. For example, the TOP factor (short for Transparency and Openness Promotion factor) has been introduced, which rates journals on openness, transparency, and preregistration policies (see Woolston, 2020; https://topfactor.org/; https://www.cos.io/ourservices/top-guidelines; see also, Munafò et al., 2017). A range of other alternative metrics, such as innovation and clarity of scientific articles have been mentioned in for example Macleod et al. (2014) and Yarkoni (2012; see Table 4). In The Leiden Manifesto for research metrics, Hicks et al. (2015) proposed to use multiple metrics to provide a better and more balanced view of the quality and value of a research paper. For other initiatives see e.g., Curry (2018). Additionally, Piwowar (2013) suggested that we should not only look at the impact of peer-reviewed articles, but also at the impact of other non-traditional scientific products such as datasets, blogs, and software, amongst others (see also, Ravenscroft et al., 2017, for a study on impact outside academia). Altmetric (altmetric.com) allows to assess broader impact of work beyond citations in peer reviewed articles, for example citations in blogs. Though this is an improvement to the IF, having impact does not necessarily mean that the work has high quality or that the impact is beneficial (as opposed to detrimental). Alternatives (or additions to) the impact factor are summarized in Table 4.

New and combined metrics might be a suitable replacement, or an addition to, more traditional citation-based metrics, though it will likely take time for them to gain value in the scientific community.

\section{Interim conclusion}

Incentives in science have mainly focused on quantity of publications, impact factors, and citations. However, none of them directly reflect scientific quality and rigor. Depending on the context, such metrics might or might not be important for the individual scientist's future career advancement. New scientific metrics (e.g., reflecting openness and transparency) have been developed that provide decent alternatives (see, Table 4). 


\begin{tabular}{|c|c|c|c|}
\hline Metric & $\begin{array}{c}\text { Objective or subjective } \\
\text { (e.g., based on graded } \\
\text { evaluation by formal } \\
\text { and informal peer } \\
\text { reviewers) }\end{array}$ & Remarks & Mentioned in \\
\hline $\begin{array}{l}\text { Transparency and } \\
\text { Openness (TOP) } \\
\text { factor }\end{array}$ & (Rather) objective & $\begin{array}{l}\text { Mainly used to rate } \\
\text { journals, but parts of it } \\
\text { could also be used to } \\
\text { rate individual papers' } \\
\text { transparency }\end{array}$ & $\begin{array}{l}\text { e.g., Woolston } \\
\text { (2020) }\end{array}$ \\
\hline
\end{tabular}

\section{General scientific Subjective quality}

Methodological Subjective
rigor

e.g., Yarkoni (2012);

Macleod et al. (2014)

\begin{tabular}{lr} 
Innovation & Subjective \\
\hline Clarity & Subjective
\end{tabular}

e.g., Yarkoni (2012)

e.g., Yarkoni (2012)

$\begin{array}{ll}\text { Wider view on the Objective } & \text { See, e.g., e.g., Piwowar } \\ \text { impact of articles } & \text { altmetric.com. Note (2013) } \\ \text { or journals (e.g., } & \text { that having impact } \\ \text { times mentioned in } & \text { does not necessarily } \\ \text { traditional media, } & \text { imply high quality nor } \\ \text { twitter, or blogs) } & \text { positive influence. }\end{array}$

A combination of Combination of objective multiple traditional and subjective evaluation and new metrics
Could include all of the e.g., Hicks et above as well as the al. (2015) traditional journal impact factor

Table 4. List of examples of alternative metrics to the traditional impact factor. Many of these could be used to rate individual articles, journals, and other materials (e.g., software code or datasets). Note that this is not a complete list of suggested alternatives, but shows the ones that are important in our view. ${ }^{1}$ Graded evaluation of quality of papers has been proposed by multiple sources, see our paragraph on alternatives to the current publication system and peer-review system. 


\section{In perspective: Science as a social enterprise}

Science is done by human beings working in groups as part of a broad scientific society. Science can thus be seen as a social enterprise (Maiväli, 2015; Munafò et al., 2017). Though this has advantages, it also comes at costs that are specific to human cognition and behavior - at the personal, group, and societal level. Learning about the influence of our human nature - and the limits of human understanding - on the way science is conducted might prevent us from major pitfalls.

The scheme of hypothetico-deductive science is hidden under a thick layer of human scientific behavior, including questionable research practices and cognitive (as well as social) biases (see, Munafò et al., 2017). QRPs such as selective reporting and $p$-hacking break the strict deductive path of hypotheses-testing aspects of science. Human traits, such as the need to belong to - and be accepted by - a social group, sensitivity to social status, social hierarchy, and personal pride, amongst many others, can diffuse and confuse scientific claims. Overinterpretation and misinterpretation of results (e.g., by supervisors or senior researchers with a specific unconscious or conscious agenda) can lead to biased conclusions. Similarly, abstracts can be used to give a positive spin to research and research results, which might be improved by structuring of, or reporting guidelines for, abstracts (Glasziou et al., 2014). In order to dig up objective knowledge underneath the layer of human behavior, one has to adhere to sound scientific principles, such as transparency, rigorousness, preregistration, moral behavior, and objectivity, amongst many others.

A scientific system that aims to make sound scientific claims should take all these human, social, and societal traits into account, and should try to either minimize the effects they exert, or to use them as a means to improve science. For example, Yarkoni (2012) suggested to associate a researcher's reputation with previously undervalued topics such as peer review, answering other scientist's questions, and data sharing; and to use online applications to foster this. Scientific reputation was also mentioned in Mambrini et al. (2020) as an incentive for high quality work. Making peer reviews citable (see, e.g., Tracz \& Lawrence, 2016), or even paying reviewers for their work (Mambrini et al., 2020), could also be seen as valuable incentives to improve peer review.

\section{Crowdsourcing and horizontal models of science}

In the third part of their series on 'scientific utopia', Uhlmann et al. (2019) argue that the current system of vertical integration, meaning that a research lab on its own goes through the whole cycle of hypothesis formulation, study design, data acquisition, data analysis, and report writing, has both benefits and disadvantages. The benefits are that this system might spark independent thinking and creativity, which might lead to novel ideas and progress. However, such a vertical model precludes shared resources, which might hamper the use of larger samples and assessing the robustness of effects (Uhlmann et al., 2019). They argue that a horizontal model of science, in which research labs carry out a specific component of the study (hence combining resources and efforts), might lead to better scientific research, and does not preclude the use of a vertical model for initial hypothesis generation. Crowdsourcing, or 'team science', has also been advocated as solution to low powered studies (Munafò et al.; 2017). In human neuroscience, initiatives such as the Human Connectome Project (see, 
https://www.humanconnectome.org/) could be seen as a step towards horizonal models of science.

\section{Conclusions}

Past research has elucidated questionable research practices (QRPs) in life and social sciences, hampering scientific quality and rigor. Estimates of results reproducibility have often been low, though sometimes moderate-to-high depending on the study design and definition of a successful reproduction. Although estimates of reproducibility have often been lower than what would be considered desirable, it is important to note that failed results (or inferential) reproducibility is not a sign that the initial research had low quality or suffered from scientific misconduct. Many factors including power, prior odds, type-I errors, design, and sample characteristics (amongst many others) could explain the failed reproduction. Poor reproducibility is however not the only reason for improvement, as previous research has shown that a lot of resources (e.g., money, effort) in biomedical science are wasted (Macleod et al., 2014), which likely extends to many disciplines within the social and life sciences.

In the recent decade, many new ideas and implementations have been suggested to improve reproducibility and scientific practice. In this paper we reviewed the literature concerning improvement of life and social sciences. With new ideas, technology, and knowledge in combination with an honest approach to discover patterns in the world, and developing theories about the world, we can vastly improve the way we conduct life sciences, social sciences, and beyond. As such, researchers should focus on aggregating effects across registered replication attempts for solid scientific knowledge, and on transparency, openness, and complete reporting of all performed analyses, data, and code. Complete openness might however be unwarranted, since eternal de-identification cannot be guaranteed. Researchers should also focus on expanding statistical practices beyond standard use of the NHST, justifying statistical choices a prior (even if they are widely used), changing incentives from quantity (e.g., number of publications or h-indices) to quality and rigor (e.g., by providing graded ratings of the article's quality by peers), and improving the current peer review system by allowing for (optionally) anonymous, continuous, graded, and open peer reviews. Largescale collaborations and horizontal models of science might be a way forward to achieve sufficient power, formulate interesting hypothesis, and incorporate different points of view.

Each new implementation will most likely come with drawbacks, which should be studied, evaluated, and dealt with in a systematic way (see also, Munafò et al., 2017). Recent findings have for example shown that preprint servers are sometimes used to disseminate low-quality studies to a wide audience, potentially having deleterious effects on important political decisions (see e.g., Besançon et al., 2020). This could be avoided by creating preprint servers that are only accessible for researchers. Other meta-scientific studies have shown that scientific self-correction is suboptimal (e.g., Greenberg, 2009), and it has been suggested that this might be improved by encouraging critical citations and (anonymous) post-publication peer review (Bordignon, 2020). Even other meta-scientific studies have shown that effect sizes of reproductions are often much smaller than the original studies, and that statistical correction methods are not able to deal with all QRPs (Kvarven et al., 2020), showing that research 
should be conducted in environments that discourage selective reporting and selective publication.

Recently, articles have criticized current meta-research, its main focus on reproducibility, and over-generalization of claims made by proponents of reproducibility research and preregistration. Devezer et al. (2020) for example called for formalism in meta-research and warned against considering reproducibility as a demarcation principle. Similarly, Szollosi et al. (2019) warned against ungrounded optimism of the influence of preregistration on scientific theory development. This debate will hopefully help to continuously finetune and improve modern scientific practices and thinking, meta-research, and formulate boundary conditions on the claims that are made in the field of meta-science.

In summary, accumulation of knowledge and applications in social and life sciences could become much more resource-efficient (time, energy), credible, and sound with new implementations (see also Macleod et al., 2014). Fanelli et al. (2015) suggested that a good way forward to prevent research misconduct is to promote transparent communication and criticism between scientists, and to improve research integrity policies. We encourage researchers to make checklists of what improvements they aim to achieve in the upcoming years (for an example, see Supplementary Figure 1). Ultimately, improving life and social sciences might require new generations of scientists to follow a full-time curriculum on research conduct, open/transparent science, statistics, methodology, and formal theory development, amongst others, which goes far beyond (but also includes) learning to share data and code, or how to preregister studies. This should be interwoven with multiple internships, should be compensated well financially (we should break with the low financial reward PhD researchers receive in many countries), and should be achieved without an extreme pressure to publish. Similar post-graduate models have been used for specialization in medicine, showing its feasibility.

\section{Limitations}

Though we did our very best to give a balanced view by incorporating a wide range of literature and different point of views, this was by definition not a systematic review. As the field of metascience grows, and the debate on good scientific practice and theory expands, future studies could do systematic literature searches, potentially guided by our review.

This review did not extensively focus on ideas on how to improve research funding. For the interested reader we would refer to Chalmers et al. (2014). The review also does not extensively include ideas on improving policy in science. We would like to refer the interested reader to Salman et al. (2014).

\section{Potential conflicts of interest}

Though we would like to start or maintain a career in academia or industry, for which an extra publication could be helpful, we do not believe that this has influenced the content nor quality of our manuscript in any way. We do not have financial or ideological conflicts of interest to disclose. 


\section{Acknowledgements}

HA was funded by the Special Research Fund of Ghent University (grant No. BOF16/DOC/282; https://www.ugent.be/), and a grant for long stay abroad from Research Foundation Flanders (FWO-V; awarded to HA; http://www.fwo.be). SVDB was supported by a grant from Research Foundation Flanders (G036716N) awarded to DM. NP was funded by the Deakin University Postgraduate Research Scholarship (DUPRS).

The authors would like to thank Prof. Dr. Tom Verguts, Prof. Dr. Beatrijs Moerkerke, and Lara Vankelecom for their valuable feedback and comments. The authors would also like to thank Dr. Regan Gallagher and Dr. Kristina Sabaroedin for interesting feedback and discussions that have contributed to the manuscript. 


\section{Supplementary Materials}

Supplementary Figure 1

\section{A checklist to improve our research}

\begin{tabular}{|c|c|}
\hline 1. Aggregate effects across replication attempts & 4. Expanding our statistical toolbox and justify choices beforehand \\
\hline $\begin{array}{l}\square \text { Reproduce a study's design, and check the aggregated effects } \\
\text { Publish it irrespective of the results (e.g., using the Registered Reports-format in the } \\
\text { next section) }\end{array}$ & $\begin{array}{l}\square \text { Focus on interpreting effect sizes and respective confidence intervals, in addition to p-values } \\
\square \text { Consider non-standard statistics when more applicable }\end{array}$ \\
\hline $\begin{array}{l}\text { 2. Achieve more solid empirical claims: } \\
\square \text { Publish a (pre)registered article }\end{array}$ & $\begin{array}{l}\text { Testing evidence in favor of the null-hypothesis (equivalence testing or Bayesian } \\
\text { statistics) } \\
\text { Bayesian statistics } \\
\text { Statistical learning }\end{array}$ \\
\hline $\begin{array}{l}\text { - The Open Science Framework (OSF; https://losf.io) } \\
\text { Registered Report (for participating journals, see: https://cos.io/rr/) }\end{array}$ & 5. Changing incentives \\
\hline 3. Transparency and openness & $\begin{array}{l}\square \text { Consider trying new publication initiatives } \\
\text { E.g., preprint reviews in eLife, publication in F1000Research }\end{array}$ \\
\hline $\begin{array}{l}\text { Publish } \square \text { data, } \square \text { all performed analyses, } \square \text { research plan, and/or } \square \text { code } \\
\text { : Publish them alongside the article (e.g., using a link to a repository or to OSF.io) } \\
\text { Publish data in a data paper (e.g.,.) }\end{array}$ & $\begin{array}{l}\text { 6. Become a continuous and open peer reviewer: } \\
\square \text { Write an open (optionally anonymous) review of (or a comment on) a preprint or peer } \\
\text { reviewed article using e.g., PubPeer (https://pubpeer.com) or a preprint server (e.g., } \\
\text { https://biorxiv.org), amongst others. }\end{array}$ \\
\hline
\end{tabular}

Supplementary Figure 1. An example of a checklist researchers could use to improve their research. 


\section{References}

Aliseda, A. (2006). Abductive Reasoning: Logical Investigations into Discovery and Explanation. Springer Netherlands. https://doi.org/10.1007/1-4020-3907-7

Alleva, L. (2006). Taking time to savour the rewards of slow science. Nature, 443(7109), 271-271. https://doi.org/10.1038/443271e

Altman, D. G. (1994). The scandal of poor medical research. BMJ, 308(6924), 283-284. https://doi.org/10.1136/bmj.308.6924.283

Amrhein, V., Greenland, S., \& McShane, B. (2019). Scientists rise up against statistical significance. Nature, 567(7748), 305-307. https://doi.org/10.1038/d41586-019-00857-9

Arns, M. (2014). Open access is tiring out peer reviewers. Nature News, 515(7528), 467. https://doi.org/10.1038/515467a

Artino, A. R. J., Driessen, E. W., \& Maggio, L. A. (2019). Ethical Shades of Gray: International Frequency of Scientific Misconduct and Questionable Research Practices in Health Professions Education. Academic Medicine, 94(1), 76-84. https://doi.org/10.1097/ACM.0000000000002412

Baker, M. (2016). 1,500 scientists lift the lid on reproducibility. Nature News, 533(7604), 452. https://doi.org/10.1038/533452a

Baker, M., \& Dolgin, E. (2017). Cancer reproducibility project releases first results. Nature News, 541(7637), 269. https://doi.org/10.1038/541269a

Banks, G. C., Rogelberg, S. G., Woznyj, H. M., Landis, R. S., \& Rupp, D. E. (2016). Editorial: Evidence on Questionable Research Practices: The Good, the Bad, and the Ugly. Journal of Business and Psychology, 31(3), 323-338. https://doi.org/10.1007/s10869-016-9456-7

Barnes, N. (2010). Publish your computer code: It is good enough. Nature, 467(7317), 753-753. https://doi.org/10.1038/467753a

Begley, C. G., \& Ellis, L. M. (2012). Raise standards for preclinical cancer research. Nature, 483(7391), 531-533. https://doi.org/10.1038/483531a

Benjamin, D. J., Berger, J. O., Johannesson, M., Nosek, B. A., Wagenmakers, E.-J., Berk, R., Bollen, K. A., Brembs, B., Brown, L., Camerer, C., Cesarini, D., Chambers, C. D., Clyde, M., Cook, T. D., De Boeck, P., Dienes, Z., Dreber, A., Easwaran, K., Efferson, C., ... Johnson, V. E. (2018). Redefine statistical significance. Nature Human Behaviour, 2(1), 6-10. https://doi.org/10.1038/s41562-017-0189-z

Besançon, L., Peiffer-Smadja, N., Segalas, C., Jiang, H., Masuzzo, P., Smout, C., Billy, E., Deforet, M., \& Leyrat, C. (2020). Open Science Saves Lives: Lessons from the COVID-19 Pandemic. BioRxiv, 2020.08.13.249847. https://doi.org/10.1101/2020.08.13.249847

Bordignon, F. (2020). Self-correction of science: A comparative study of negative citations and post-publication peer review. Scientometrics, 124(2), 1225-1239. https://doi.org/10.1007/s11192-020-03536-z

Bornmann, L., Mutz, R., \& Daniel, H.-D. (2010). A Reliability-Generalization Study of Journal Peer Reviews: A Multilevel Meta-Analysis of Inter-Rater Reliability and Its Determinants. PLoS ONE, 5(12). https://doi.org/10.1371/journal.pone.0014331

Bossuyt, P. M., Reitsma, J. B., Bruns, D. E., Gatsonis, C. A., Glasziou, P. P., Irwig, L. M., Lijmer, J. G., Moher, D., Rennie, D., \& de Vet, H. C. W. (2003). Towards Complete and Accurate 
Reporting of Studies of Diagnostic Accuracy: The STARD Initiative. Annals of Internal Medicine, 138(1), 40-44. https://doi.org/10.7326/0003-4819-138-1-200301070-00010

Botvinik-Nezer, R., Holzmeister, F., Camerer, C. F., Dreber, A., Huber, J., Johannesson, M., Kirchler, M., Iwanir, R., Mumford, J. A., Adcock, R. A., Avesani, P., Baczkowski, B. M., Bajracharya, A., Bakst, L., Ball, S., Barilari, M., Bault, N., Beaton, D., Beitner, J., ... Schonberg, T. (2020). Variability in the analysis of a single neuroimaging dataset by many teams. Nature, 1-7. https://doi.org/10.1038/s41586-020-2314-9

Bravo, G., Grimaldo, F., López-Iñesta, E., Mehmani, B., \& Squazzoni, F. (2019). The effect of publishing peer review reports on referee behavior in five scholarly journals. Nature Communications, 10(1), 322. https://doi.org/10.1038/s41467-018-08250-2

Breeze, J. L., Poline, J.-B., \& Kennedy, D. N. (2012). Data sharing and publishing in the field of neuroimaging. GigaScience, 1(1). https://doi.org/10.1186/2047-217X-1-9

Brown, G. G., Mathalon, D. H., Stern, H., Ford, J., Mueller, B., Greve, D. N., McCarthy, G., Voyvodic, J., Glover, G., Diaz, M., Yetter, E., Burak Ozyurt, I., Jorgensen, K. W., Wible, C. G., Turner, J. A., Thompson, W. K., \& Potkin, S. G. (2011). Multisite Reliability of Cognitive BOLD Data. Neurolmage, 2163-2175. https://doi.org/10.1016/j.neuroimage.2010.09.076

Button, K. S., loannidis, J. P. A., Mokrysz, C., Nosek, B. A., Flint, J., Robinson, E. S. J., \& Munafò, M. R. (2013). Power failure: Why small sample size undermines the reliability of neuroscience. Nature Reviews Neuroscience, 14(5), 365-376. https://doi.org/10.1038/nrn3475

Bzdok, D. (2017). Classical Statistics and Statistical Learning in Imaging Neuroscience. Frontiers in Neuroscience, 11. https://doi.org/10.3389/fnins.2017.00543

Camerer, C. F., Dreber, A., Holzmeister, F., Ho, T.-H., Huber, J., Johannesson, M., Kirchler, M., Nave, G., Nosek, B. A., Pfeiffer, T., Altmejd, A., Buttrick, N., Chan, T., Chen, Y., Forsell, E., Gampa, A., Heikensten, E., Hummer, L., Imai, T., ... Wu, H. (2018). Evaluating the replicability of social science experiments in Nature and Science between 2010 and 2015. Nature Human Behaviour, 2(9), 637-644. https://doi.org/10.1038/s41562-018-0399-z

Campbell, E. G., Clarridge, B. R., Gokhale, M., Birenbaum, L., Hilgartner, S., Holtzman, N. A., \& Blumenthal, D. (2002). Data Withholding in Academic Genetics: Evidence From a National Survey. JAMA, 287(4), 473-480. https://doi.org/10.1001/jama.287.4.473

Carp, J. (2012). On the Plurality of (Methodological) Worlds: Estimating the Analytic Flexibility of fMRI Experiments. Frontiers in Neuroscience, 6. https://doi.org/10.3389/fnins.2012.00149

Carter, E. C., Schönbrodt, F. D., Gervais, W. M., \& Hilgard, J. (2019). Correcting for Bias in Psychology: A Comparison of Meta-Analytic Methods. Advances in Methods and Practices in Psychological Science, 2(2), 115-144. https://doi.org/10.1177/2515245919847196

Casey, B. J., Cohen, J. D., O'Craven, K., Davidson, R. J., Irwin, W., Nelson, C. A., Noll, D. C., Hu, X., Lowe, M. J., Rosen, B. R., Truwitt, C. L., \& Turski, P. A. (1998). Reproducibility of fMRI Results across Four Institutions Using a Spatial Working Memory Task. Neurolmage, 8(3), 249-261. https://doi.org/10.1006/nimg.1998.0360

Center for Open Science (n. d.). Open Science Badges enhance openness, a core value of scientific practice. Center for Open Science. Retrieved June $4^{\text {th }}, 2020$, from: https://www.cos.io/our-services/badges

Chambers, C. D., Feredoes, E., Muthukumaraswamy, S. D., \& Etchells, P. J. (2014). Instead of "playing the game" it is time to change the rules: Registered Reports at AIMS Neuroscience 
and beyond. AIMS Neuroscience 2014, Vol. 1, Pages 4-17. https://doi.org/10.3934/Neuroscience.2014.1.4

Chalmers, I., Bracken, M. B., Djulbegovic, B., Garattini, S., Grant, J., Gülmezoglu, A. M., Howells, D. W., loannidis, J. P. A., \& Oliver, S. (2014). How to increase value and reduce waste when research priorities are set. The Lancet, 383(9912), 156-165. https://doi.org/10.1016/S01406736(13)62229-1

Chan, A.-W., Hróbjartsson, A., Haahr, M. T., Gøtzsche, P. C., \& Altman, D. G. (2004). Empirical evidence for selective reporting of outcomes in randomized trials: Comparison of protocols to published articles. JAMA, 291(20), 2457-2465. https://doi.org/10.1001/jama.291.20.2457

Chan, A.-W., Song, F., Vickers, A., Jefferson, T., Dickersin, K., Gøtzsche, P. C., Krumholz, H. M., Ghersi, D., \& van der Worp, H. B. (2014). Increasing value and reducing waste: Addressing inaccessible research. The Lancet, 383(9913), 257-266. https://doi.org/10.1016/S01406736(13)62296-5

Cooper, G. S., \& Meterko, V. (2019). Cognitive bias research in forensic science: A systematic review. Forensic Science International, 297, 35-46. https://doi.org/10.1016/j.forsciint.2019.01.016

Cumming, G. (2014). The New Statistics: Why and How. Psychological Science, 25(1), 7-29. https://doi.org/10.1177/0956797613504966

Curry, S. (2018). Let's move beyond the rhetoric: It's time to change how we judge research. Nature, 554(7691), 147-147. https://doi.org/10.1038/d41586-018-01642-w

Day, R. (2020, November 21). 15 Topics to Consider as You Review Code in Data Science. Towards data science. https://towardsdatascience.com/15-topics-to-consider-as-youreview-code-in-data-science-10eff0182e71

de Montjoye, Y.-A. de, Radaelli, L., Singh, V. K., \& Pentland, A. "Sandy." (2015). Unique in the shopping mall: On the reidentifiability of credit card metadata. Science, 347(6221), 536-539. https://doi.org/10.1126/science.1256297

Devezer, B., Navarro, D., Vandekerckhove, J., \& Buzbas, E. (2020). The case for formal methodology in scientific reform. Biorxiv, 048306. https://www.biorxiv.org/content/10.1101/2020.04.26.048306v2

de Winter, J., \& Happee, R. (2013). Why Selective Publication of Statistically Significant Results Can Be Effective. PLoS ONE, 8(6). https://doi.org/10.1371/journal.pone.0066463

Directorate-General for Research and Innovation (European Commission, 2018, November 26). Turning FAIR into reality. Final Report and Action Plan from the European Commission Expert Group on FAIR Data. Luxembourg: Publications office of the European Union, 2018.

Dreber, A., Pfeiffer, T., Almenberg, J., Isaksson, S., Wilson, B., Chen, Y., Nosek, B., \& Johannesson, M. (2015). Using prediction markets to estimate the reproducibility of scientific research. PNAS, 112(50), 15343-15347.

Eglen, S. J., Marwick, B., Halchenko, Y. O., Hanke, M., Sufi, S., Gleeson, P., Silver, R. A., Davison, A. P., Lanyon, L., Abrams, M., Wachtler, T., Willshaw, D. J., Pouzat, C., \& Poline, J.-B. (2017). Toward standard practices for sharing computer code and programs in neuroscience. Nature Neuroscience, 20(6), 770-773. https://doi.org/10.1038/nn.4550

Eisner, D. A. (2018). Reproducibility of science: Fraud, impact factors and carelessness. Journal of Molecular and Cellular Cardiology, 114, 364-368. https://doi.org/10.1016/j.yjmcc.2017.10.009 
Eklund, A., Nichols, T. E., \& Knutsson, H. (2016). Cluster failure: Why fMRI inferences for spatial extent have inflated false-positive rates. Proceedings of the National Academy of Sciences, 113(28), 7900-7905. https://doi.org/10.1073/pnas.1602413113

Esteban, O., Birman, D., Schaer, M., Koyejo, O. O., Poldrack, R. A., \& Gorgolewski, K. J. (2017). MRIQC: Advancing the automatic prediction of image quality in MRI from unseen sites. PLoS ONE, 12(9), e0184661. https://doi.org/10.1371/journal.pone.0184661

Esteban, O., Markiewicz, C. J., Blair, R. W., Moodie, C. A., Isik, A. I., Erramuzpe, A., Kent, J. D., Goncalves, M., DuPre, E., Snyder, M., Oya, H., Ghosh, S. S., Wright, J., Durnez, J., Poldrack, R. A., \& Gorgolewski, K. J. (2019). fMRIPrep: A robust preprocessing pipeline for functional MRI. Nature Methods, 16(1), 111-116. https://doi.org/10.1038/s41592-018-02354

Etz, A., \& Vandekerckhove, J. (2016). A Bayesian Perspective on the Reproducibility Project: Psychology. PLOS ONE, 11(2), e0149794. https://doi.org/10.1371/journal.pone.0149794

Eva, K. W. (2017). How would you like your salami? A guide to slicing. Medical Education, 51(5), 456-457. https://doi.org/10.1111/medu.13285

Fanelli, D. (2009). How Many Scientists Fabricate and Falsify Research? A Systematic Review and Meta-Analysis of Survey Data. PLOS ONE, 4(5), e5738. https://doi.org/10.1371/journal.pone.0005738

Fanelli, D. (2010). Do Pressures to Publish Increase Scientists' Bias? An Empirical Support from US States Data. PLoS ONE, 5(4), e10271. https://doi.org/10.1371/journal.pone.0010271

Fanelli, D. (2018). Opinion: Is science really facing a reproducibility crisis, and do we need it to? Proceedings of the National Academy of Sciences, 115(11), 2628-2631. https://doi.org/10.1073/pnas.1708272114

Fanelli, D., Costas, R., \& Larivière, V. (2015). Misconduct Policies, Academic Culture and Career Stage, Not Gender or Pressures to Publish, Affect Scientific Integrity. PLOS ONE, 10(6), e0127556. https://doi.org/10.1371/journal.pone.0127556

Fernandes, J. D., Sarabipour, S., Smith, C. T., Niemi, N. M., Jadavji, N. M., Kozik, A. J., Holehouse, A. S., Pejaver, V., Symmons, O., Bisson Filho, A. W., \& Haage, A. (2020). A survey-based analysis of the academic job market. ELife, 9, e54097. https://doi.org/10.7554/eLife.54097

Fiedler, K. (2017). What Constitutes Strong Psychological Science? The (Neglected) Role of Diagnosticity and A Priori Theorizing. Perspectives on Psychological Science, 12(1), 46-61. https://doi.org/10.1177/1745691616654458

Fiedler, K., Kutzner, F., \& Krueger, J. I. (2012). The Long Way From a-Error Control to Validity Proper: Problems With a Short-Sighted False-Positive Debate. Perspectives on Psychological Science, 7(6), 661-669. https://doi.org/10.1177/1745691612462587

Filevich, E., Lisofsky, N., Becker, M., Butler, O., Lochstet, M., Martensson, J., Wenger, E., Lindenberger, U., \& Kühn, S. (2017). Day2day: Investigating daily variability of magnetic resonance imaging measures over half a year. BMC Neuroscience, 18(1), 65. https://doi.org/10.1186/s12868-017-0383-y

Forstmeier, W., Wagenmakers, E.-J., \& Parker, T. H. (2017). Detecting and avoiding likely falsepositive findings - a practical guide. Biological Reviews, 92(4), 1941-1968. https://doi.org/10.1111/brv.12315

Foster, E. D., \& Deardorff, A. (2017). Open Science Framework (OSF). Journal of the Medical Library Association : JMLA, 105(2), 203-206. https://doi.org/10.5195/jmla.2017.88 
Freedman, L. P., Cockburn, I. M., \& Simcoe, T. S. (2015). The Economics of Reproducibility in $\begin{array}{llll}\text { Preclinical Research. PLoS Biology, 13(6), } & \text { e1002165. }\end{array}$ https://doi.org/10.1371/journal.pbio.1002165

Friedman, L., Stern, H., Brown, G. G., Mathalon, D. H., Turner, J., Glover, G. H., Gollub, R. L., Lauriello, J., Lim, K. O., Cannon, T., Greve, D. N., Bockholt, H. J., Belger, A., Mueller, B., Doty, M. J., He, J., Wells, W., Smyth, P., Pieper, S., ... Potkin, S. G. (2007). Test-retest and between-site reliability in a multicenter fMRI study. Human Brain Mapping, 29(8), 958-972. https://doi.org/10.1002/hbm.20440

Friesike, S., \& Schildhauer, T. (2015). Open science: Many good resolutions, very few incentives, yet. In I. M. Welpe, J., Wollersheim, S., Ringelhan, M., \& Osterloh (Eds.) Incentives and Performance: Governance of Research Organizations (pp. 277-289). Berlin/Heidelberg: Springer, Cham.

Friston, K. J., Harrison, L., \& Penny, W. (2003). Dynamic causal modelling. Neurolmage, 19(4), 1273-1302. https://doi.org/10.1016/s1053-8119(03)00202-7

Fu, D. Y., \& Hughey, J. J. (2019). Releasing a preprint is associated with more attention and citations for the peer-reviewed article. ELife, 8, e52646. https://doi.org/10.7554/eLife.52646

Gewin, V. (2016). Data sharing: An open mind on open data. Nature, 529(7584), 117-119. https://doi.org/10.1038/nj7584-117a

Glasziou, P., Altman, D. G., Bossuyt, P., Boutron, I., Clarke, M., Julious, S., Michie, S., Moher, D., \& Wager, E. (2014). Reducing waste from incomplete or unusable reports of biomedical research. The Lancet, 383(9913), 267-276. https://doi.org/10.1016/S0140-6736(13)62228$\mathrm{X}$.

Glasziou, P. P., Sanders, S., \& Hoffmann, T. (2020). Waste in covid-19 research. BMJ (Clinical Research Ed.), 369, m1847. https://doi.org/10.1136/bmj.m1847

Gilbert, D. T., King, G., Pettigrew, S., \& Wilson, T. D. (2016). Comment on "Estimating the reproducibility of psychological science." Science, 351(6277), 1037-1037. https://doi.org/10.1126/science.aad7243

Glatard, T., Lewis, L. B., Ferreira da Silva, R., Adalat, R., Beck, N., Lepage, C., Rioux, P., Rousseau, M.-E., Sherif, T., Deelman, E., Khalili-Mahani, N., \& Evans, A. C. (2015). Reproducibility of neuroimaging analyses across operating systems. Frontiers in Neuroinformatics, 9. https://doi.org/10.3389/fninf.2015.00012

Goldbeck-Wood, S. (1999). Evidence on peer review-Scientific quality control or smokescreen? BMJ : British Medical Journal, 318(7175), 44-45.

Goodman, S. N., Fanelli, D., \& Ioannidis, J. P. A. (2016). What does research reproducibility mean? Science Translational Medicine, 8(341), 341ps12-341ps12. https://doi.org/10.1126/scitransImed.aaf5027

Gorgolewski, K. J., Auer, T., Calhoun, V. D., Craddock, R. C., Das, S., Duff, E. P., Flandin, G., Ghosh, S. S., Glatard, T., Halchenko, Y. O., Handwerker, D. A., Hanke, M., Keator, D., Li, X., Michael, Z., Maumet, C., Nichols, B. N., Nichols, T. E., Pellman, J., ... Poldrack, R. A. (2016). The brain imaging data structure, a format for organizing and describing outputs of $\begin{array}{lllll}\text { neuroimaging experiments. Scientific Data, } & 3(1),\end{array}$ https://doi.org/10.1038/sdata.2016.44

Gorgolewski, K. J., Margulies, D. S., \& Milham, M. P. (2013). Making Data Sharing Count: A Publication-Based Solution. Frontiers in Neuroscience, 7. https://doi.org/10.3389/fnins.2013.00009 
Gorgolewski, K. J., \& Poldrack, R. A. (2016). A Practical Guide for Improving Transparency and Reproducibility in Neuroimaging Research. PLOS Biology, 14(7), e1002506. https://doi.org/10.1371/journal.pbio.1002506

Gotzsche, P. C. (1987). Reference bias in reports of drug trials. BMJ, 295(6599), 654-656. https://doi.org/10.1136/bmj.295.6599.654

Greenberg, S. A. (2009). How citation distortions create unfounded authority: Analysis of a citation network. The BMJ, 339. https://doi.org/10.1136/bmj.b2680

Hanson, B., Sugden, A., \& Alberts, B. (2011). Making Data Maximally Available. Science, 331(6018), 649-649. https://doi.org/10.1126/science.1203354

Head, M. L., Holman, L., Lanfear, R., Kahn, A. T., \& Jennions, M. D. (2015). The Extent and Consequences of P-Hacking in Science. PLoS Biology, 13(3), e1002106. https://doi.org/10.1371/journal.pbio.1002106

Hicks, D., Wouters, P., Waltman, L., de Rijcke, S., \& Rafols, I. (2015). Bibliometrics: The Leiden Manifesto for research metrics. Nature News, 520(7548), 429. https://doi.org/10.1038/520429a

Ioannidis, J. P. A. (2005). Why Most Published Research Findings Are False. PLoS medicine, 2(8): e124. https://doi.org/10.1371/journal.pmed.0020124.

loannidis, J. P. A. (2012). Why Science Is Not Necessarily Self-Correcting. Perspectives on Psychological Science, 7(6), 645-654. https://doi.org/10.1177/1745691612464056

Ioannidis, J. P. A., Fanelli, D., Dunne, D. D., \& Goodman, S. N. (2015). Meta-research: Evaluation and Improvement of Research Methods and Practices. PLoS Biology, 13(10), e1002264. https://doi.org/10.1371/journal.pbio.1002264

John, L. K., Loewenstein, G., \& Prelec, D. (2012). Measuring the Prevalence of Questionable Research Practices With Incentives for Truth Telling. Psychological Science, 23(5), 524532. https://doi.org/10.1177/0956797611430953

Jones, C. W., Keil, L. G., Holland, W. C., Caughey, M. C., \& Platts-Mills, T. F. (2015). Comparison of registered and published outcomes in randomized controlled trials: A systematic review. BMC Medicine, 13, 282. https://doi.org/10.1186/s12916-015-0520-3

Kidwell, M. C., Lazarević, L. B., Baranski, E., Hardwicke, T. E., Piechowski, S., Falkenberg, L.-S., Kennett, C., Slowik, A., Sonnleitner, C., Hess-Holden, C., Errington, T. M., Fiedler, S., \& Nosek, B. A. (2016). Badges to Acknowledge Open Practices: A Simple, Low-Cost, Effective Method for Increasing Transparency. PLoS Biology, 14(5), e1002456. https://doi.org/10.1371/journal.pbio.1002456

Klein, R. A., Ratliff, K. A., Vianello, M., Adams, R. B., Bahník, Š., Bernstein, M. J., Bocian, K., Brandt, M. J., Brooks, B., Brumbaugh, C. C., Cemalcilar, Z., Chandler, J., Cheong, W., Davis, W. E., Devos, T., Eisner, M., Frankowska, N., Furrow, D., Galliani, E. M., ... Nosek, B. A. (2014). Investigating Variation in Replicability: A "Many Labs" Replication Project. Social Psychology, 45(3), 142-152. https://doi.org/10.1027/1864-9335/a000178

Klein, R. A., Vianello, M., Hasselman, F., Adams, B. G., Adams, R. B., Alper, S., Aveyard, M., Axt, J. R., Babalola, M. T., Bahník, Š., Batra, R., Berkics, M., Bernstein, M. J., Berry, D. R., Bialobrzeska, O., Binan, E. D., Bocian, K., Brandt, M. J., Busching, R., ... Nosek, B. A. (2018). Many Labs 2: Investigating Variation in Replicability Across Samples and Settings. Advances in Methods and Practices in Psychological Science, 1(4), 443-490. https://doi.org/10.1177/2515245918810225 
Kong, X.-Z., Group, E. L. W., \& Francks, C. (2019). An illustration of reproducibility in neuroscience research in the absence of selective reporting. BioRxiv, 866301. https://doi.org/10.1101/866301

Kvarven, A., Strømland, E., \& Johannesson, M. (2020). Comparing meta-analyses and preregistered multiple-laboratory replication projects. Nature Human Behaviour, 4(4), 423434. https://doi.org/10.1038/s41562-019-0787-z

Lakens, D. (2016, February 14). Why you don't need to adjust your alpha level for all tests you'll do in your lifetime. [Blog Post]. Retrieved from http://daniellakens.blogspot.com/2016/02.

Lakens, Daniel, Adolfi, F. G., Albers, C. J., Anvari, F., Apps, M. A. J., Argamon, S. E., Baguley, T., Becker, R. B., Benning, S. D., Bradford, D. E., Buchanan, E. M., Caldwell, A. R., Van Calster, B., Carlsson, R., Chen, S.-C., Chung, B., Colling, L. J., Collins, G. S., Crook, Z., .. Zwaan, R. A. (2018). Justify your alpha. Nature Human Behaviour, 2(3), 168-171. https://doi.org/10.1038/s41562-018-0311-x

Lakens, D., Scheel, A. M., \& Isager, P. M. (2018). Equivalence Testing for Psychological Research: A Tutorial. Advances in Methods and Practices in Psychological Science, 1(2), 259-269. https://doi.org/10.1177/2515245918770963

Lawrence, R. (2020, March 25). F1000 Research Ltd wins European Commission contract to set up an open access publishing platform [Blog post]. Retrieved from https://blog.f1000.com/2020/03/25/f1000-research-Itd-wins-european-commission-contractto-set-up-an-open-access-publishing-platform/

Lee, M. D., Criss, A. H., Devezer, B., Donkin, C., Etz, A., Leite, F. P., Matzke, D., Rouder, J. N., Trueblood, J., White, C., \& Vandekerckhove, J. (2019). Robust modeling in cognitive science [Preprint]. PsyArXiv. https://doi.org/10.31234/osf.io/dmfhk

Locke, E. A. (2007). The Case for Inductive Theory Building. Journal of Management, 33(6), 867890. https://doi.org/10.1177/0149206307307636

Macleod, M. R., Michie, S., Roberts, I., Dirnagl, U., Chalmers, I., loannidis, J. P. A., Salman, R. A.-S., Chan, A.-W., \& Glasziou, P. (2014). Biomedical research: Increasing value, reducing waste. The Lancet, 383(9912), 101-104. https://doi.org/10.1016/S0140-6736(13)62329-6

McShane, B. B., Gal, D., Gelman, A., Robert, C., \& Tackett, J. L. (2019). Abandon Statistical Significance. The American Statistician, 73(sup1), 235-245. https://doi.org/10.1080/00031305.2018.1527253

Magee, A. F., May, M. R., \& Moore, B. R. (2014). The Dawn of Open Access to Phylogenetic Data. PLOS ONE, 9(10), e110268. https://doi.org/10.1371/journal.pone.0110268

Mambrini, A., Baronchelli, A., Starnini, M., Marinazzo, D., \& De Domenico, M. (2020). PRINCIPIA: A Decentralized Peer-Review Ecosystem. ArXiv:2008.09011. http://arxiv.org/abs/2008.09011

Maiväli, Ü. (2015). Chapter 7-Science as a Social Enterprise. In Ü. Maiväli (Ed.), Interpreting Biomedical Science (pp. 291-336). Academic Press. https://doi.org/10.1016/B978-0-12418689-7.00007-7

Moher, D., Liberati, A., Tetzlaff, J., Altman, D. G., \& Group, T. P. (2009). Preferred Reporting Items for Systematic Reviews and Meta-Analyses: The PRISMA Statement. PLOS Medicine, 6(7), e1000097. https://doi.org/10.1371/journal.pmed.1000097

Munafò, M., Nosek, B., Bishop, D. Button, K., Chambers, C., Percie du Sert, N., ..., \& loannidis, J. P. A. (2017). A manifesto for reproducible science. Nature Human Behavior, 1, 0021. https://doi.org/10.1038/s41562-016-0021 
Muthukrishna, M., \& Henrich, J. (2019). A problem in theory. Nature Human Behaviour, 3(3), 221-229. https://doi.org/10.1038/s41562-018-0522-1

Narayanan, A., \& Shmatikov, V. (2008). Robust De-anonymization of Large Sparse Datasets. 2008 IEEE Symposium on Security and Privacy (Sp 2008), 111-125. https://doi.org/10.1109/SP.2008.33

Nelson, L. D., Simmons, J., \& Simonsohn, U. (2018). Psychology's Renaissance. Annual Review of Psychology, 69(1), 511-534. https://doi.org/10.1146/annurev-psych-122216-011836

Neuroskeptic (2015, April). P-values and exploratory research. Discover. Retrieved from https://www.discovermagazine.com/mind/p-values-and-exploratory-research.

Nichols, T. E., Das, S., Eickhoff, S. B., Evans, A. C., Glatard, T., Hanke, M., Kriegeskorte, N., Milham, M. P., Poldrack, R. A., Poline, J.-B., Proal, E., Thirion, B., Van Essen, D. C., White, T., \& Yeo, B. T. T. (2017). Best practices in data analysis and sharing in neuroimaging using MRI. Nature Neuroscience, 20(3), 299-303. https://doi.org/10.1038/nn.4500

Nickerson (1998). Confirmation Bias: A Ubiquitous Phenomenon in Many Guises. Review of General Psychology, 2(2), 175-220. doi:10.1037/1089-2680.2.2.175

Nosek, B. A., \& Bar-Anan, Y. (2012). Scientific Utopia: I. Opening Scientific Communication. Psychological Inquiry, 23(3), 217-243. https://doi.org/10.1080/1047840X.2012.692215

Nosek, B. A., Ebersole, C. R., DeHaven, A. C., \& Mellor, D. T. (2018). The preregistration revolution. Proceedings of the National Academy of Sciences, 115(11), 2600-2606. https://doi.org/10.1073/pnas.1708274114

Nosek, B. A., \& Errington, T. M. (2017). Making sense of replications. ELife, 6, e23383. https://doi.org/10.7554/eLife.23383

Nosek, B. A., \& Lakens, D. (2014). Registered reports: A method to increase the credibility of published results. Social Psychology. Hogrefe and Huber Publishers. https://doi.org/10.1027/1864-9335/a000192

Nosek, B. A., Spies, J. R., \& Motyl, M. (2012). Scientific Utopia: II. Restructuring Incentives and Practices to Promote Truth Over Publishability. Perspectives on Psychological Science, 7(6), 615-631. https://doi.org/10.1177/1745691612459058

Nuzzo, R. (2014). Scientific method: Statistical errors. Nature News, 506(7487), 150. https://doi.org/10.1038/506150a

Nuzzo, R. (2015). How scientists fool themselves - and how they can stop. Nature News, 526(7572), 182. https://doi.org/10.1038/526182a

Open Science Collaboration (2015). Estimating the reproducibility of psychological science. Science, 349(6251). https://doi.org/10.1126/science.aac4716

Perneger, T. V. (1998). What's wrong with Bonferroni adjustments. BMJ : British Medical Journal, 316(7139), 1236-1238.

Plesser, H. E. (2017). Reproducibility vs. Replicability: A Brief History of a Confused Terminology. Frontiers in Neuroinformatics, 11. https://doi.org/10.3389/FNINF.2017.00076

Piwowar, H. A., Day, R. S., \& Fridsma, D. B. (2007). Sharing Detailed Research Data Is Associated with Increased Citation Rate. PLoS ONE, 2(3). https://doi.org/10.1371/journal.pone.0000308 
Poldrack, R. A., Baker, C. I., Durnez, J., Gorgolewski, K. J., Matthews, P. M., Munafò, M. R., Nichols, T. E., Poline, J.-B., Vul, E., \& Yarkoni, T. (2017). Scanning the horizon: Towards transparent and reproducible neuroimaging research. Nature Reviews Neuroscience, 18(2), 115-126. https://doi.org/10.1038/nrn.2016.167

Poldrack, R. A., Feingold, F., Frank, M. J., Gleeson, P., de Hollander, G., Huys, Q. J. M., Love, B. C., Markiewicz, C. J., Moran, R., Ritter, P., Rogers, T. T., Turner, B. M., Yarkoni, T., Zhan, M., \& Cohen, J. D. (2019). The Importance of Standards for Sharing of Computational Models and Data. Computational Brain \& Behavior, 2(3), 229-232. https://doi.org/10.1007/s42113-019-00062-x

Poldrack, R., Fletcher, P., Henson, R., Worsley, K., Brett, M. and Nichols, T. (2008). Guidelines for reporting an fMRI study. Neurolmage, 40(2), pp.409-414.

Prasad, V., Vandross, A., Toomey, C., Cheung, M., Rho, J., Quinn, S., Chacko, S. J., Borkar, D., Gall, V., Selvaraj, S., Ho, N., \& Cifu, A. (2013). A Decade of Reversal: An Analysis of 146 Contradicted Medical Practices. Mayo Clinic Proceedings, 88(8), 790-798. https://doi.org/10.1016/j.mayocp.2013.05.012

Prinz, F., Schlange, T., \& Asadullah, K. (2011). Believe it or not: How much can we rely on published data on potential drug targets? Nature Reviews Drug Discovery, 10(9), 712-712. https://doi.org/10.1038/nrd3439-c1

Quintana, D. S., \& Williams, D. R. (2018). Bayesian alternatives for common null-hypothesis significance tests in psychiatry: A non-technical guide using JASP. BMC Psychiatry, 18(1), 178. https://doi.org/10.1186/s12888-018-1761-4

Ravenscroft, J., Liakata, M., Clare, A., \& Duma, D. (2017). Measuring scientific impact beyond academia: An assessment of existing impact metrics and proposed improvements. PLOS ONE, 12(3), e0173152. https://doi.org/10.1371/journal.pone.0173152

Refining our policies on co-authorship requirements [editorial]. Sci Data 4, 170133 (2017). https://doi.org/10.1038/sdata.2017.133

Rowhani-Farid, A., Allen, M., \& Barnett, A. G. (2017). What incentives increase data sharing in health and medical research? A systematic review. Research Integrity and Peer Review, 2(1), 4. https://doi.org/10.1186/s41073-017-0028-9

Rubin, M (2017). Do p Values Lose Their Meaning in Exploratory Analyses? It Depends How You Define the Familywise Error Rate. Review of General Psychology, 21(3), 269-275. https://doi.org/10.1037/gpr0000123

Salman, R. A.-S., Beller, E., Kagan, J., Hemminki, E., Phillips, R. S., Savulescu, J., Macleod, M., Wisely, J., \& Chalmers, I. (2014). Increasing value and reducing waste in biomedical research regulation and management. The Lancet, 383(9912), 176-185. https://doi.org/10.1016/S0140-6736(13)62297-7

Schoenbrodt, F. (2015). Questionable research practices [PowerPoint slides]. Retrieved from https://osf.io/bh7zv/.

Schoenbrodt (2018). Changing incentive structures to foster the actual sharing rate of open data [PowerPoint slides]. Retrieved from https://www.open-science-conference.eu/wpcontent/uploads/2018/03/OSC2018_Schoenbrodt.pdf

Schmucker, C., Schell, L. K., Portalupi, S., Oeller, P., Cabrera, L., Bassler, D., Schwarzer, G., Scherer, R. W., Antes, G., Elm, E. von, Meerpohl, J. J., \& Consortium, on behalf of the O. (2014). Extent of Non-Publication in Cohorts of Studies Approved by Research Ethics 
Committees or Included in Trial Registries. PLOS ONE, 9(12), e114023. https://doi.org/10.1371/journal.pone.0114023

Simmons, J. P., Nelson, L. D., \& Simonsohn, U. (2011). False-Positive Psychology: Undisclosed Flexibility in Data Collection and Analysis Allows Presenting Anything as Significant. Psychological Science, 22(11), 1359-1366. https://doi.org/10.1177/0956797611417632

Soderberg, C. K., Errington, T. M., \& Nosek, B. A. (2020). Credibility of preprints: An interdisciplinary survey of researchers. Royal Society Open Science, 7(10), 201520. https://doi.org/10.1098/rsos.201520

Spector, P. E., Rogelberg, S. G., Ryan, A. M., Schmitt, N., \& Zedeck, S. (2014). Moving the Pendulum Back to the Middle: Reflections on and Introduction to the Inductive Research Special Issue of Journal of Business and Psychology. Journal of Business and Psychology, 29(4), 499-502. https://doi.org/10.1007/s10869-014-9372-7

Stern, B. M., \& O'Shea, E. K. (2019). A proposal for the future of scientific publishing in the life sciences. PLOS Biology, 17(2), e3000116. https://doi.org/10.1371/journal.pbio.3000116

Stigbrand, T. (2017). Retraction Note to multiple articles in Tumor Biology. Tumor Biology. https://doi.org/10.1007/s13277-017-5487-6

Stodden, V., Seiler, J., \& Ma, Z. (2018). An empirical analysis of journal policy effectiveness for computational reproducibility. Proceedings of the National Academy of Sciences, 115(11), 2584-2589. https://doi.org/10.1073/pnas.1708290115

Szollosi, A., Kellen, D., Navarro, D., Shiffrin, R., van Rooij, I., Van Zandt, T., \& Donkin, C. (2019). Is preregistration worthwhile? Trends in Cognitive Sciences, 24 (2), 94-95. https://doi.org/10.1016/j.tics.2019.11.009

Szucs, D., \& loannidis, J. (2017a). Empirical assessment of published effect sizes and power in the recent cognitive neuroscience and psychology literature. PLoS Biology, 15(3), e2000797. https://doi.org/10.1371/journal.pbio.2000797

Szucs, D., \& loannidis, J. P. A. (2017b). When Null Hypothesis Significance Testing Is Unsuitable for Research: A Reassessment. Frontiers in Human Neuroscience, 11. https://doi.org/10.3389/fnhum.2017.00390

The Society for Research in Child Development (2019, April 1). Policy on Scientific Integrity, Transparency, and Openness. Retrieved from: https://www.srcd.org/policy-scientificintegrity-transparency-and-openness

Thompson, W. H., Wright, J., Bissett, P. G., \& Poldrack, R. A. (2020). Dataset decay and the problem of sequential analyses on open datasets. ELife, 9, e53498. https://doi.org/10.7554/eLife.53498

Tijdink, J. K., Verbeke, R., \& Smulders, Y. M. (2014). Publication Pressure and Scientific Misconduct in Medical Scientists. Journal of Empirical Research on Human Research Ethics, 9(5), 64-71. https://doi.org/10.1177/1556264614552421

Tracz, V., \& Lawrence, R. (2016). Towards an open science publishing platform [version 1; $\begin{array}{llll}\text { referees: } & 2 & \text { approved]. } & \text { F1000Research, }\end{array}$ https://doi.org/10.12688/f1000research.7968.1

Turner, E. H., Matthews, A. M., Linardatos, E., Tell, R. A., \& Rosenthal, R. (2008). Selective Publication of Antidepressant Trials and Its Influence on Apparent Efficacy. New England Journal of Medicine, 358(3), 252-260. https://doi.org/10.1056/NEJMsa065779

Uhlmann EL, Ebersole CR, Chartier CR, et al. Scientific Utopia III: Crowdsourcing Science. Perspectives on Psychological Science, 14(5), 711-733. doi:10.1177/1745691619850561 
Ulrich, R., \& Miller, J. (2020). Questionable research practices may have little effect on replicability. ELife, 9, e58237. https://doi.org/10.7554/eLife.58237

van Dijk, D., Manor, O., \& Carey, L. B. (2014). Publication metrics and success on the academic job market. Current Biology, 24(11), R516-R517. https://doi.org/10.1016/j.cub.2014.04.039

van Panhuis, W. G., Paul, P., Emerson, C., Grefenstette, J., Wilder, R., Herbst, A. J., Heymann, D., \& Burke, D. S. (2014). A systematic review of barriers to data sharing in public health. BMC Public Health, 14(1), 1144. https://doi.org/10.1186/1471-2458-14-1144

Vesper, I. (2018). Peer reviewers unmasked: Largest global survey reveals trends. Nature. https://doi.org/10.1038/d41586-018-06602-y

Wagenmakers, E.-J. (2007). A practical solution to the pervasive problems of $p$ values. Psychonomic Bulletin \& Review, 14(5), 779-804. https://doi.org/10.3758/BF03194105

Welcome to a new ERA of reproducible publishing (2020, August 24). ELife; eLife Sciences Publications Limited. https://elifesciences.org/labs/dc5acbde/welcome-to-a-new-era-ofreproducible-publishing

Wen, H., Wang, H.-Y., He, X., \& Wu, C.-I. (2018). On the low reproducibility of cancer studies. National Science Review, 5(5), 619-624. https://doi.org/10.1093/nsr/nwy021

Whitacker, K. (2008). Barriers to reproducible research (and how to overcome them) [PowerPoint Slides]. Retrieved from: https://figshare.com/articles/Barriers_to_reproducible_research_and_how_to_overcome_th em_/7140050

Wicherts, J. M., Borsboom, D., Kats, J., \& Molenaar, D. (2006). The poor availability of psychological research data for reanalysis. American Psychologist, 61(7), 726-728. https://doi.org/10.1037/0003-066X.61.7.726

Wilkinson, M. D., Dumontier, M., Aalbersberg, Ij. J., Appleton, G., Axton, M., Baak, A., Blomberg, N., Boiten, J.-W., da Silva Santos, L. B., Bourne, P. E., Bouwman, J., Brookes, A. J., Clark, T., Crosas, M., Dillo, I., Dumon, O., Edmunds, S., Evelo, C. T., Finkers, R., ... Mons, B. (2016). The FAIR Guiding Principles for scientific data management and stewardship. Scientific Data, 3. https://doi.org/10.1038/sdata.2016.18

Wilson, B. M., \& Wixted, J. T. (2018). The Prior Odds of Testing a True Effect in Cognitive and Social Psychology. Advances in Methods and Practices in Psychological Science, 1(2), 186-197. https://doi.org/10.1177/2515245918767122

Woolston (2020, February 18). TOP Factor rates journals on transparency, openness. Nature index. https://www.natureindex.com/news-blog/top-factor-rates-journals-on-transparencyopenness

Yarkoni, T. (2012). Designing next-generation platforms for evaluating scientific output: What scientists can learn from the social web. Frontiers in Computational Neuroscience, 6. https://doi.org/10.3389/fncom.2012.00072

Yarkoni, T. (2018, October 2). No, it's not The Incentives-it's you [Blog post]. Retrieved from: https://www.talyarkoni.org/blog/2018/10/02/no-its-not-the-incentives-its-you/

Yarkoni, T. (2019a, July 13). I hate open science [Blog post]. Retrieved from: https://www.talyarkoni.org/blog/2019/07/13/i-hate-open-science/

Yarkoni, T. (2019b, November 22). The Generalizability Crisis. PsyArXiv. https://doi.org/10.31234/osf.io/jaw35 
Yeung, A. W. K. (2017). Do Neuroscience Journals Accept Replications? A Survey of Literature. Frontiers in Human Neuroscience, 11. https://doi.org/10.3389/fnhum.2017.00468 